Universidade de São Paulo

Instituto de Física

\title{
Aproximação Tempo de Relação: Modelos Alternativos em Teoria \\ Cinética Clássica
}

\author{
Diego Sales de Oliveira
}

Orientador: José Ademir Sales de Lima

Dissertação de mestrado apresentada ao Instituto de Física para a obtenção do título de Mestre em Ciências

Banca Examinadora:

Prof. Dr. José Ademir Sales de Lima (IAG-USP)

Prof. Dr. Silvio Roberto de Azevedo Salinas (IFUSP)

Prof. Dr. Raimundo Silva Junior (UFRN)

São Paulo 




\section{Agradecimentos}

À minha família;

Á minha companheira Amanda Horta de Lima;

Ao orientador Prof. Dr. José Ademir Sales de Lima pela sugestão do tema, e pelo apoio e estímulo na realização deste projeto;

Aos Profs. Dr. Vilson Tonin Zanchin e Dr. Francisco Eugênio Mendonça da Silveira, meus orientadores de iniciação científica durante a gradução;

Ao CNPq, pelo apoio financeiro;

Ao Instituto de Física da Universidade de São Paulo por fornecer o suporte necessário para o desenvolvimento do projeto. 

"A liberdade é para a ciência o que o ar é para o animal." Jules Henri Poincaré

"O binômio de Newton é tão belo como a Vênus de Milo."

Fernando Pessoa 



\section{Resumo}

As possíveis soluções da equação integro-diferencial de Boltzmann constituem uma importante ferramenta para o estudo de gases e plasmas. No entanto, suas soluções analíticas são difíceis de serem encontradas. Uma abordagem bastante utilizada na literatura para obter soluções aproximadas da equação de Boltzmann é através de hipóteses que simplificam a forma da integral de colisão.

Nesta dissertação, discutimos dois modelos colisionais alternativos que generalizam o método originalmente proposto por Bhatnagar, Gross e Krook, usualmente referido na literatura como aproximação BGK. O primeiro deles é um modelo de relaxação de segunda ordem, no qual introduzimos um segundo tempo de relaxação, $\tau_{2}$, relacionado com efeitos não lineares. O segundo modelo é baseado em outra generalização do modelo BGK obtida através de uma lei de potência parametrizada por um índice $\alpha$. No limite $\alpha \rightarrow 1$ o modelo BGK padrão é recuperado.

As duas aproximações são fisicamente interpretadas. Além disso, para ilustrar nossos resultados com algumas aplicações mais quantitativas, obtemos as expressões analíticas para diversos coeficientes de transporte,a saber: a condutividade térmica $(\kappa)$, a viscosidade de cisalhamento $(\eta)$ e a condutividade elétrica $(\sigma)$. Em particular, no modelo de relaxação de segunda ordem, as correções dependem da razão $\tau_{1} / \tau_{2}$, onde $\tau_{1}$ é a escala de tempo característica do modelo BGK padrão e $\tau_{2}$ a nova escala de tempo associada aos efeitos não

lineares. Finalmente, como um resultado geral, mostramos também que todas as correções nos coeficientes de transporte dependem numa certa potência do chamado número de Knudsen. 



\section{Abstract}

The possible solutions of the integro-differential Boltzmann equation constitute an important tool for studying gases and plasmas. However, its analytical solutions are hardly derived. An approach often adopted in the literature for obtaining approximate solutions of the Boltzmann equation is to consider some simplifying hypothesis on the collisional term.

In this Dissertation, we discuss two different alternative collisional models which generalize the method originally proposed by Bhatnagar, Gross e Krook, and usually referred to as BGK approximation. The first one is a second order relaxation model in which a second relaxation time, $\tau_{2}$, related with the nonlinear effects, is introduced. The second one is based on a different generalization of the BGK model which is obtained through a power law parameterized by a index $\alpha$. In the limit $\alpha \rightarrow 1$, the BGK model is recovered. Both approximations are physically interpreted. Further, in order to illustrate our results with some more quantitative applications, we derive the analytical expressions for several transport coefficients, among them: the thermal conductivity $(\kappa)$, the shear viscosity $(\eta)$ and the electric conductivity $(\sigma)$. In particular, for the second order relaxation model, we find that the corrections depend on the ratio $\tau_{1} / \tau_{2}$ where $\tau_{1}$ is the characteristic time scale of the BGK model and $\tau_{2}$ describe the nonlinear effects. Finally, as a general result, it is also shown that all the corrections on the transport coefficients depend on a given power of the so-called Knudsen number. 



\section{Lista de Figuras}

2.1 Variação de Entropia . . . . . . . . . . . . . . . . . . . . . 29

3.1 Diagrama de Colisões . . . . . . . . . . . . . . . . . . . . . . . 40

3.2 Cilindro de Colisões . . . . . . . . . . . . . . . . . . . . . . . . 42

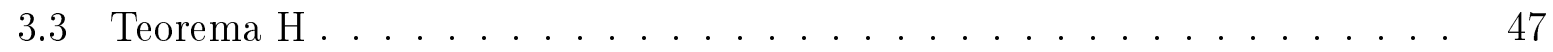

3.4 Representação de um gás dotado de um gradiente de velocidade . . . . . . . 54

4.1 Decaimento para o Equiíbrio na Aproximação de Segunda Ordem . . . . . 60

4.2 Decaimento para o Equilíbrio . . . . . . . . . . . . . . . . 71

4.3 Condutividade Elétrica Segundo a Lei de Potências . . . . . . . . . . . . 73 



\section{Sumário}

1. Introdução . . . . . . . . . . . . . . . . . . . . . . . . 17

2. Termodinâmica de não-Equilíbrio . . . . . . . . . . . . . . . . . . . . . . . . 23

2.1 Introdução . . . . . . . . . . . . . . . . . . . . . . . 23

2.2 Equações de Balanço . . . . . . . . . . . . . . . . . . . . . . . . . 24

2.2.1 Equação de Balanço de Massa . . . . . . . . . . . . . . . . . . . . . 24

2.2.2 Balanço de Momento e Energia Mecânica . . . . . . . . . . . . . . . 26

2.2.3 Equação de Balanço e Primeira Lei da Termodinâmica . . . . . . . 27

2.2.4 Balanço de Entropia . . . . . . . . . . . . . . . . . . . . . . 28

2.2.5 Segunda Lei da Termodinâmica e Balanço de Entropia . . . . . . . 28

2.2.6 A Fonte de Entropia . . . . . . . . . . . . . . . . . . . . . . . 30

2.3 Positividade dos Coeficientes das Equações Fenomenológicas . . . . . . . . 32

2.3.1 Fluidos na Presença de um Campo Elétrico . . . . . . . . . . . . . 33

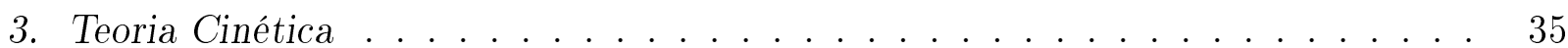

3.1 Introdução . . . . . . . . . . . . . . . . . . . . . . . 35

3.2 Função de Distribuição de Velocidades . . . . . . . . . . . . . . . . . . 36

3.2.1 A Derivação de Maxwell . . . . . . . . . . . . . . . . . . . . . 36

3.2 .2 Forma Local da Distribuição de Maxwell . . . . . . . . . . . . . . . 38

3.3 Equação de Boltzmann . . . . . . . . . . . . . . . . . . . . . . . . 39

3.3.1 Integral de Colisões de Boltzmann . . . . . . . . . . . . . . . . . . . 41

3.3.2 Teorema H de Boltzmann . . . . . . . . . . . . . . . . . . 44

3.3.3 Teorema H e a Distribuição Maxwelliana . . . . . . . . . . . . . . . . 47

3.3.4 Aproximação Tempo de Relaxação . . . . . . . . . . . . . . . . . . 48 
3.4 Cálculo dos Coeficientes de Transporte . . . . . . . . . . . . . . . . 50

3.4.1 Equação de Boltzmann Perturbada . . . . . . . . . . . . . . 51

3.4 .2 Condutividade Térmica . . . . . . . . . . . . . . . . 51

3.4.3 Viscosidade ....................... 53

3.4 .4 Condutividade Elétrica . . . . . . . . . . . . . . . . 55

3.4.5 Razão de Eucken . . . . . . . . . . . . . . . . . . . 56

4. Modelos Colisionais Alternativos . . . . . . . . . . . . . . . . 57

4.1 Introdução . . . . . . . . . . . . . . . . . . 57

4.2 Modelo Colisional com Termo de Segunda Ordem . . . . . . . . . . . . . 57

4.3 Calculo dos Coeficientes de Transporte . . . . . . . . . . . . . . 60

4.3.1 Equação de Boltzmann Perturbada . . . . . . . . . . . . . . 60

4.3.2 Condutividade Térmica . . . . . . . . . . . . . 62

4.3.3 Viscosidade . . . . . . . . . . . . . . . . . . . . . 64

4.3.4 Condutividade elétrica . . . . . . . . . . . . . 66

4.3.5 Razão de Eucken e Correções de Segunda Ordem . . . . . . . . . . 68

4.4 Modelo Colisional Tipo Lei de Potência . . . . . . . . . . . . . . . . . . . . 69

4.5 Cálculo da Condutividade Elétrica . . . . . . . . . . . . . . . 71

5. Conclusões e Perspectivas . . . . . . . . . . . . . . . . . . 75

$\begin{array}{ll}\text { Apêndice } & 79\end{array}$

A. Elementos de Volume no Espaço de Fase - Invariância . . . . . . . . . . . . . . . 81

B. Decomposição do Produto Tensorial (Tensor de Viscosidade) . . . . . . . . . . . 83

C. Equações de Balanço . . . . . . . . . . . . . . . . . . . . . . . 85

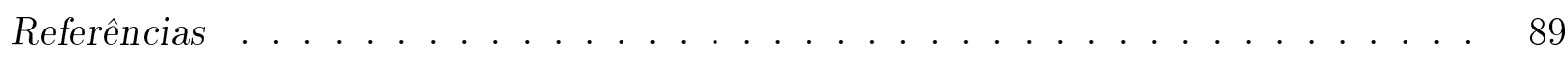


Capítulo 1

\section{Introdução}

É de grande interesse em Física ou mesmo na área de Engenharia o estudo dos chamados sistemas ou meios contínuos. Tais sistemas são dotados de propriedades físicas expressas através de variáveis contínuas e definidas para cada ponto do sistema e instante de tempo. Essas variáveis expressam médias macroscópicas locais cujas características e possíveis comportamentos a nível microscópico podem ser ignoradas [1, 2]. Uma característica interessante dos sistemas contínuos é a capacidade de serem indefinidamente fracionados. Isto ocorre porque todo elemento fracionado ainda conterá um grande número de átomos ou moléculas que constituem o sistema macroscópico. Este procedimento é satisfatório quando as dimensões do material em questão (seus comprimentos característicos macroscópicos) são muito maiores que os comprimentos característicos microscópicos (livre caminho médio, distâncias interatômicas ou intermoleculares, etc) [1,3]. Isto significa que os elementos de volumes locais possuem um grande número de partículas (átomos ou moléculas).

O tratamento macroscópico é bastante empregado para sistemas contínuos tratados pela dinâmica dos fluidos, ou seja, no estudo do comportamento térmico e dinâmico de líquidos e gases (a propriedade macroscópica básica distinguindo líquidos e gases é a compressibilidade). Para fluidos perfeitos, por exemplo, o estado de movimento do meio é descrito através de 5 variáveis contínuas ou campos independentes, a saber: as 3 componentes do campo de velocidades do fluido, $\mathbf{v}(\mathbf{r}, t)$, mais duas variáveis termodinâmicas independentes, tais como, a densidade $\rho(\mathbf{r}, t)$ e a pressão $p(\mathbf{r}, t)[4]$. Na chamada descrição euleriana, que será adotada aqui (ver capítulo 1), o campo de velocidades é calculado em um ponto fixo no espaço e não no sistema de referência co-móvel com um determinado elemento de volume do fluido (descrição lagrangiana).

Será a descrição macroscópica sempre consistente? Para exemplificar considere o caso 
de um gás. Localmente, uma partícula do gás atravessará em média uma distância típica $\lambda$ (livre caminho médio), antes de modificar seu estado de movimento ao colidir com outras partículas. O efeito estatístico das colisões é distribuir a velocidade das partículas em torno de uma velocidade média local do gás. Se estivermos interessados no que acontece em escalas $L>>\lambda$, poderemos imaginar o gás como um sistema contínuo com velocidade, densidade, pressão e temperatura, em geral, funções da posição e do tempo. No entanto, muitas vezes os efeitos cinéticos desempenham um papel especial devido ao grande número de colisões elásticas e inelásticas, ionizações e diversas interações entre partículas e campos externos, tal como ocorre com íons em campos eletromagnéticos [5] ou gases sob ação de campos gravitacionais [6]. Em tais casos, as equações macroscópicas não podem prever grandes variações em escalas da ordem de $\lambda$. Nessas circunstâncias, para se ter uma descrição mais precisa, é necessário utilizar uma teoria microscópica, tal como a chamada teoria cinética dos gases, ou simplesmente, teoria cinética $[3,7,8,9]$.

A descrição microscópica da dinâmica de um gás a partir da chamada teoria cinética tem tido um notável sucesso no tratamento de gases diluídos. Um exemplo bastante conhecido está associado com a determinação dos chamados coeficientes de transporte. Pela teoria de fluidos fora de equilíbrio termodinâmico, sabemos que os coeficientes de transporte (condutividade térmica, viscosidade volumar, viscosidade de shear e condutividade elétrica) devem ser positivos definidos, tal como exigido pela segunda lei da termodinâmica (a fonte de entropia é positivo definida). No entanto, a dependência matemática de tais coeficientes nas variáveis básicas (temperatura, pressão, livre tempo médio, etc), geralmente determinada com base em procedimentos experimentais, pode ser prevista apenas no âmbito da teoria cinética.

De grande importância na formulação da teoria cinética é a função de distribuição, $f(\mathbf{r}, \mathbf{v}, t)$. Tal função representa a densidade de pontos no espaço de fase e a partir dela, podemos determinar as variáveis macroscópicas médias como pressão e densidade [7, 8, 10, 11]. A função de distribuição, bem como sua evolução temporal, é obtida através da chamada equação de Boltzmann, uma equação integro-diferencial que determina a evolução temporal de $f(\mathbf{r}, \mathbf{v}, t)$ e, consequentemente, a evolução das variáveis básicas do sistema. Sua parte integral (o termo colisional) determina as variações sofridas por $f$ no espaço de fase devido às colisões entre as partículas, sendo usualmente conhecida por integral de colisões de Boltzmann [8, 12]. 
O termo colisional presente na equação de Boltzmann é de fundamental importância para os fenômenos de transporte, uma vez que é através das colisões que ocorre o transporte das propriedades macroscópicas como massa, momento e energia $[11,12]$. Contudo a integral de colisões é difícil de ser resolvida. De fato, apenas nos casos mais simples, ou sob certas aproximações, suas soluções analíticas podem ser explicitamente obtidas $[7,8,13]$

É bem conhecido que as dificuldades de se resolver a equação de Boltzmann completa pode ser evitada através de algumas aproximações no termo colisional. Uma abordagem frequentemente utilizada e que proporciona uma boa precisão no tratamento cinético das colisões é usualmente referida como aproximação tempo de relaxação, originalmente proposta por Bhatnagar, Gross e Krook [14, 15]. No chamado model BGK, a complexidade dos cálculos que seriam geradas pela integral de colisões é evitada substituindo-se o termo colisional por uma aproximação linear que não apenas facilita os cálculos, mas também permite uma compreensão física bastante simples da tendência do sistema atingir o estado de equilíbrio termodinâmico.

Embora fornecendo uma aproximação razoável para diversas quantidades físicas quando os gradientes das forças termodinâmicas são pequenos, é de se esperar que muitos fenômenos de interesse físico não sejam descritos corretamente pelo simplificado modelo BGK. Alguns exemplos envolvem tratamento de ondas choque, comportamento de plasmas na presença de intensos campos magnéticos, processos de reconexão magnética, ou mesmo o cálculo de coeficientes de transporte quando os gradientes são moderamente intensos [8]. Em tais casos, para uma descrição mais precisa dos processos físicos, é interessante obter soluções numéricas da equação de Boltzmann completa ou propor modelos alternativos que generalizem a método BGK padrão. É nesse contexto que observamos a importância de modelos aproximativos mais precisos para resolver a Equação de Boltzmann. De fato, ao se evitar tanto quanto possível o tratamento numérico, podemos obter uma compreensão mais simples da física subjacente as situações mais complexas geralmente tratadas através de métodos numéricos ou observadas em simulações de N-corpos.

Recentemente, o modelo BGK foi adotado para recalcular os coeficientes cinéticos supondo que a distribuıcão de equilíbrio é descrita por uma lei de potência (q-maxwelliana) proposta no âmbito da chamada teoria cinética não extensiva [16, 17, 18]. Expressões analíticas foram obtidas para os coeficientes de viscosidade de cisalhamento (shear viscos- 
ity) e condutividade térmica [19]. A solução da equação de Boltzmann para um plasma neutro mostrou também que a condutividade elétrica não é modificada em comparação com o tratamento baseado na distribuição de Maxwell. Finalmente, mostrou-se também que a chamada razão de Eucken [20] pode limitar o parâmetro não extensivo $q$ sobre o intervalo $0.74 \leq q \leq 1$, sendo portanto marginalmente compatível com uma distribuição maxwelliana.

Nesta monografia seguimos uma linha de investigação distinta. Adotando como solução de equilíbrio a distribuição maxwelliana, propomos duas possíveis generalizações para o método proposto por Bhatnagar, Gross e Krook [14, 15]. Como veremos, tal como ocorre na abordagem BGK, as generalizações propostas são também dotadas de simples interpretação física no tocante à evolução para o equilíbrio termodinâmico. Contudo, diferente do modelo BGK padrão, existem correções para os coeficientes de transporte que dependem dos gradientes das quantidades básicas.

As linhas linhas gerais de desenvolvimento do presente trabalho estão apresentadas a seguir:

No Capítulo 2, fazemos uma breve revisão da chamada termodinâmica de não-equilíbrio. Deduzimos as equações de balanço para massa, momento e energia e apresentamos as equações que descrevem a dinâmica e a termodinâmica de fluidos simples e misturas. Discutimos o chamado princípio de entropia, calculamos a fonte de entropia em função das forças e dos fluxos termodinâmicos e, finalmente, demonstramos a positividade dos chamados coeficientes de transporte.

No Capítulo 3 dicutimos a teoria cinética de gases e mostramos algumas aplicações básicas. O conceito de função de distribuição é introduzido e a equação de Boltzmann completa, ou seja, incluindo a integral de colisões, é derivada. A partir da equação de Boltzman e da definição cinética de entropia, apresentamos uma prova do teorema $\mathrm{H}$. Finalmente, discutimos o modelo BGK e calculamos os coeficientes de transporte das equações fenomenológicas com base nessa aproximação. Expressões analíticas para a condutividade térmica $(\kappa)$, viscosidade $(\eta)$ e condutividade elétrica $(\sigma)$ são explicitamente deduzidas. De posse dos coeficientes, também calculamos a chamada relação de Eucken, uma importante quantidade experimental (sem dimensão) que relaciona a condutividade térmica com a viscosidade.

No Capítulo 4, propomos dois novos modelos colisionais alternativos que generalizam 
o método BGK e calculamos os coeficientes de transporte. Inicialmente apresentamos um modelo de relaxação de segunda ordem e soluções analíticas corrigidas para os coeficientes de transporte $(\kappa, \eta, \sigma)$ no capítulo 2 , além de discutir as correções para a relação de Eucken. Posteriormente apresentamos um modelo do tipo lei de potência, onde é calculada a condutividade elétrica $\sigma$, para exemplificar uma previsão do modelo.

Nas conclusões, apresentamos um resumo dos principais resultados e perspectivas que possibilitam futuras extensões e aplicações para o presente trabalho.

No apêndice A, demonstramos a invariância (sob transformações de coordenadas) dos elementos de volume no espaço de fase 6-D [12]. Este resultado é utilizado na transformação das integrais do termo colisional demonstradas no Capítulo 2. No apêndice B, discutimos a divisão do tensor de pressão para uma melhor compreensão das diferentes contribuições dos coeficientes de viscosidade de cisalhamento (shear viscosity) e volumar (bulk viscosity). Finalmente, no apêndice $\mathrm{C}$, fazemos uma breve discussão acerca das chamadas equações de balanço, geralmente utilizadas ao se descrever os processos de transporte.

Objetivando o auxílio em futuras consultas por parte de pesquisadores e estudantes interessados no assunto, mencionamos que o nosso contato com a literatura indica que as contribuições originais dessa monografia encontram-se no capítulo 4. Tais resultados serão brevemente submetidos a publicação [21, 22]. 
Capítulo 2

\section{Termodinâmica de não-Equilíbrio}

\subsection{Introdução}

As primeiras considerações teóricas sobre a descrição de processos termodinâmicos irreversíveis constam nos artigos de Clausius e W. Thomson (Lord Kelvin) [23]. Deste então o progresso foi considerável com os trabalho de Clausius, Duhem, Natanson, Jaumann, Lohr, Eckart e Meixner procurando estabelecer uma expressão para o variação local de entropia em sistemas não uniformes [24]. As relações de reciprocidade de Onsager [25], conectando os coeficientes nas equações fenomenológicas de transporte lineares com a descrição de processos irreversíveis e, finalmente, Prigogine [26], unindo a teoria dos processos irreversíveis e o cálculo explícito das chamadas fontes de entropia. Os trabalhos da chamada escola de Bruxelas formalizaram a chamada termodinâmica de processores irreversíveis ou termodinâmica de não-equilíbrio e sua base cinética (ver [24] e referências lá citadas).

A termodinâmica de não-equilíbrio pode ser vista como a descrição macroscópica dos processos fora do equilíbrio termodinâmico [24, 27]. Nesse sentido a termodinâmica de equilíbrio deve ser chamada de termostática. A descrição fora do equilíbrio é feita por meio de equações diferenciais parciais, onde são usadas variáveis contínuas no espaço e tempo de modo semelhante á abordagem usada nas equações que descrevem a mecânica dos fluidos e a eletrodinâmica de Maxwell.

Entre as equações de balanço que descrevem o sistema destaca-se, em particular, a do balanço de entropia. Esta equação contém o termo de criação de entropia (fonte) devido aos processos irreversíveis que ocorrem no interior do sistema [24, 27]. Podemos citar como exemplos as reações químicas e o transporte de massa, momento e(ou) energia. É desta relação entre as fontes locais de entropia e os fluxos termodinâmicos irreversíveis 
(incluindo o fluxo de entropia através da superfície) que é possível provar a positividade dos coeficientes de transporte das equações fenomenológicas [25].

Neste capítulo apresentaremos a dedução da equação do balanço de entropia, determinaremos detalhadamente o termo de fonte de entropia e a positividade dos coeficientes de transporte das equações fenomenológicas como originalmente proposto nas equações de Fourier, Navier-Stokes, lei de Ohm e na chamada lei de Fick.

\subsection{Equações de Balanço}

\subsubsection{Equação de Balanço de Massa}

Vamos considerar um sistema de volume $V$ fixo no espaço, delimitado pela superfície $\Omega$, preenchido por um fluido de única componente e densidade $\rho$ e $\mathbf{v}$ a chamada velocidade hidrodinâmica, v, isto é, a velocidade média do fluido determinada em um ponto do sistema [3]. Tanto $\rho$ quanto v podem ser, no caso mais geral, funções da posição e do tempo. Desta forma a variação de massa no interior do volume pode ser escrita como:

$$
\int_{V} \frac{\partial \rho}{\partial t} d V=-\int_{\boldsymbol{\Omega}} \rho \mathbf{v} \cdot d \boldsymbol{\Omega}+\int_{V} M d V
$$

onde $M$ é uma fonte (ou sumidouro) de massa e $d \boldsymbol{\Omega}$ o elemento de área infinitesimal, orientado para fora do elemento de volume $V$ e cujo módulo representa um elemento de área infinitesimal desta superfície.

Usando o teorema de Gauss em (2.1) podemos escrever sua forma diferencial:

$$
\frac{\partial \rho}{\partial t}=-\nabla \cdot(\rho \mathbf{v})+M
$$

Segue do apêndice B que a equação acima é a chamada equação de balanço de massa. O lado esquerdo da equação representa a variação local de massa, sendo o lado direito composto pelos termos de fluxo (descritos pelos divergentes) e de fontes (ou sumidouros).

Pela equação (2.2) é possível obter facilmente a equação para a conservação de massa, basta tomarmos $M=0$, o que nos leva a:

$$
\frac{\partial \rho}{\partial t}+\nabla \cdot(\rho \mathbf{v})=0
$$


A equação para a conservação de massa pode ser escrita de forma alternativa. Para isto vamos utilizar o conceito de derivada material ou substancial. Sendo a densidade função da posição e do tempo, $\rho(\mathbf{r}, t)$, sua variação temporal total é escrita da seguinte forma:

$$
\frac{d \rho(\mathbf{r}, t)}{d t}=\frac{\partial \rho}{\partial t}+\frac{\partial \rho}{\partial \mathbf{r}} \frac{d \mathbf{r}}{d t}
$$

Uma vez que o termo $d \mathbf{r} / d t$ é a velocidade hidrodinâmica $\mathbf{v}$, podemos reescrever a equação anterior como:

$$
\frac{d \rho}{d t}=\frac{\partial \rho}{\partial t}+\mathbf{v} \cdot \nabla \rho
$$

Assim, para qualquer quantidade física podemos definir o seguinte operador:

$$
\frac{d}{d t}=\frac{\partial}{\partial t}+\mathbf{v} \cdot \nabla
$$

Aplicando o conceito de derivada material na equação (2.3) encontramos:

$$
\frac{d \rho}{d t}=-\rho \boldsymbol{\nabla} \cdot \mathbf{v}
$$

Esta equação nos mostra que a variação de massa com relação ao tempo em um determinado volume $V$, na ausência de termos de fontes (ou sumidouros), é igual ao fluxo de massa que entra (ou sai) do sistema.

Note também que para uma propriedade arbitrária $\rho \psi$ podemos escrever:

$$
\rho \frac{d \phi}{d t}=\frac{\partial \phi \rho}{\partial t}+\nabla(\phi \rho \mathbf{v})
$$

onde foi feito o uso da equação para a conservação da massa expressa em (2.7).

Embora a discussão presente nesta seção seja feita para um fluido de única componente, seria possível tratarmos de um fluido composto de várias componentes. Podemos encontrar este tramento em "Non-Equilibrium Thermodynamics" [24].Como feito até aqui, seguiremos considerando um fluido de única componente, visto que a complexidade inserida através do tratamento multicomponente é desnecessária para a demonstração da positividade dos coeficientes de transporte, nosso objetivo com este capítulo. 


\subsubsection{Balanço de Momento e Energia Mecânica}

A equação de movimento para um fluido sob a ação de uma força externa pode ser escrita como [4]:

$$
\rho\left(\frac{\partial \mathbf{v}}{\partial t}+(\mathbf{v} \cdot \nabla) \mathbf{v}\right)=-\nabla \cdot P+\rho \mathbf{F}
$$

onde $\mathbf{v}$ é a velocidade do fluido; P é o tensor de pressão do meio (assumido simétrico $\left.P_{\alpha \beta}=P_{\beta \alpha}\right)$ e $\mathbf{F}$ a força por unidade de massa exercida sob o fluido.

O tensor pressão $P$ pode ser visto como o resultado das interações de curto alcance das partículas que compõem o sistema [24], já o termo $\mathbf{F}$ contêm as forças externas e de longo alcance atuantes no sistema [27]. Usando a relação (2.8) reescrevemos a equação de movimento (2.9) como:

$$
\frac{\partial \rho \mathbf{v}}{\partial t}=-\nabla \cdot(\rho \mathbf{v} \mathbf{v}+\mathbf{P})+\rho \mathbf{F}
$$

Esta é a equação para o balanço de momento [24, 27], onde $\rho \mathbf{v}$ é a variação local de momento, $\rho \mathbf{v v}+\mathrm{P}$ é o fluxo de momento e $\rho \mathbf{F}$ a de fonte de momento. O termo $\mathbf{F}$ representa um grande número de forças que podem atuar no sistema, entre elas forças não conservativas como a força de Lorentz. Traremos aqui somente de forças conservativas, isto é, forças deverivadas de um potencial escalar $\psi$ e independentes do tempo:

$$
\mathbf{F}=-\nabla \psi(\mathbf{r}) \quad \partial \psi / \partial t=0
$$

Antes de obtermos a equação de balanço da energia mecânica é necessário encontrar as equações de o balanço para a energia cinética e energia potencial. A equação de balanço da energia cinética é facilmente obtida, basta multiplicarmos (2.9) por v e usar a relação dada em (2.8), assim encontramos:

$$
\frac{\partial}{\partial t}\left(\frac{\rho \mathbf{v}^{2}}{2}\right)=-\boldsymbol{\nabla} \cdot\left(\frac{\rho \mathbf{v}^{2} \mathbf{v}}{2}+\mathrm{P} . \mathbf{v}\right)+\mathrm{P}: \boldsymbol{\nabla} \mathbf{v}+\mathbf{J} . \mathbf{F}
$$

onde $\mathbf{J}=\rho \mathbf{v}$ é o fluxo de massa.

Multiplicando o balanço de massa (2.2) por $\psi$ e usando as condição dadas em (2.11), encontramos a equação de balanço para a energia potencial:

$$
\frac{\partial \rho \psi}{\partial t}=-\nabla \cdot(\rho \psi \mathbf{v})-\mathbf{J} . \mathbf{F}
$$


Com as equações de balanço para a energia cinética e potencial finalmente podemos encontrar a equação de balanço de energia mecânica. Para isso basta somarmos as equações (2.12) e (2.13), o que nos leva a:

$$
\frac{\partial}{\partial t}\left(\rho\left(\frac{\mathbf{v}^{2}}{2}+\psi\right)\right)=-\boldsymbol{\nabla} \cdot\left(\rho\left(\frac{\mathbf{v}^{2}}{2}+\psi\right) \mathbf{v}+\mathrm{P} \cdot \mathbf{v}\right)+\mathrm{P}: \boldsymbol{\nabla} \mathbf{v}
$$

É possível notar pela equação acima que a energia mecânica não se conserva devido ao termo de fonte $\boldsymbol{\nabla} \mathbf{v}: \mathbf{P}$ (cuja origem última pode ser associada a viscosidade).

\subsubsection{Equação de Balanço e Primeira Lei da Termodinâmica}

O enunciado do princípio da conservação de energia nos diz: a energia de um sistema contido em um volume arbitrário $V$ se altera quando uma porção de energia flui (para dentro ou para fora) através de sua superfície $\Omega$, ou quando há produção de energia (fontes) [24]. Uma vez que não há produção, a conservação de energia é dada como:

$$
\frac{d}{d t} \int_{V} \rho e d V=\int_{V} \frac{\partial \rho e}{\partial t} d V=-\int_{\boldsymbol{\Omega}} \mathbf{J}_{e} \cdot d \boldsymbol{\Omega}
$$

onde $e$ é a energia total por unidade de massa, ou energia especifica, e $\mathbf{J}_{e}$ é o fluxo de energia total por unidade de área por unidade de tempo. Usando o teorema de Gauss encontra-se a forma diferencial (ou local) da conservação da energia total:

$$
\frac{\partial \rho e}{\partial t}=-\nabla . \mathbf{J}_{e}
$$

A energia específica $e$ é composta pela soma de todas as energias presentes no sistema, no caso a energia mecânica e interna específica, $e=\frac{1}{2} \rho \mathbf{v}^{2}+\rho \psi+u$. Em analogia a energia mecânica, o fluxo de energia total $\mathbf{J}_{e}$ é composto por uma parte convectiva, $\rho e \mathbf{v}$, relativa aos fluxos de corrente e difusão de partículas e um fluxo de energia, P.v, relativo ao trabalho mecânico que atua sob o sistema. Devemos também incluir o fluxo de calor $\mathbf{J}_{q}$, relacionado as diferenças de temperatura que eventualmente podem existir no fluido de modo a alterar u. A partir destas considerações escrevemos o fluxo de energia total como:

$$
\mathbf{J}_{e}=\rho e \mathbf{v}+\mathrm{P} \cdot \mathbf{v}+\mathbf{J}_{q} .
$$


Subtraindo (2.14) de (2.16) e usando (2.17) e a definição de energia interna específica $e$, obtemos uma expresssão para o balanço de energia interna:

$$
\frac{\partial \rho u}{\partial t}=-\nabla \cdot\left(\rho u \mathbf{v}+\mathbf{J}_{q}\right)-\mathrm{P}: \nabla \mathbf{v}
$$

A equação de balanço acima ainda pode ser escrita de uma outra maneira. Vamos começar separando o tensor de pressão em duas partes, uma escalar, a pressão hidrostática $p$, e outra tensorial $\Pi$, escrevendo $\mathrm{P}$ como $\mathrm{P}=p 1+\Pi$. Substituindo esta separação e utilizando a relação (2.8) em (2.18), encontramos:

$$
\rho \frac{d u}{d t}=-\nabla . \mathbf{J}_{q}-p \boldsymbol{\nabla} \cdot \mathbf{v}-\boldsymbol{\Pi}: \nabla \mathbf{v}
$$

Usando a definição do fluxo de calor [4]:

$$
\rho \frac{d q}{d t}=-\nabla \cdot \mathbf{J}_{q}
$$

onde $q$ é a quantidade calor por unidade de massa adicionado ao sistema e a equação (2.7) para a conservação de massa. Sendo o volume específico dado por $v=\rho^{-1}$, obtemos "a primeira lei da termodinâmica" fora do equilíbrio:

$$
\frac{d u}{d t}=\frac{d q}{d t}-p \frac{d v}{d t}-v \boldsymbol{\Pi}: \nabla \mathbf{v}
$$

\subsubsection{Balanço de Entropia}

\subsubsection{Segunda Lei da Termodinâmica e Balanço de Entropia}

No equilíbrio termodinâmico é possível definir uma função de estado chamada entropia, $S$, determinada a partir de parâmetros extensivos do sistema. A variação de entropia do sistema corresponde á soma de dois termos:

$$
d S=d S_{i}+d S_{e}
$$

onde $d S_{i}$ é a entropia gerada no sistema e $d S_{e}$ a variação de entropia devido ao fluxo de $S$ através da fronteira (ver figura 2.1).

Pela segunda lei da termodinâmica em um sistema isolado a variação de entropia é 


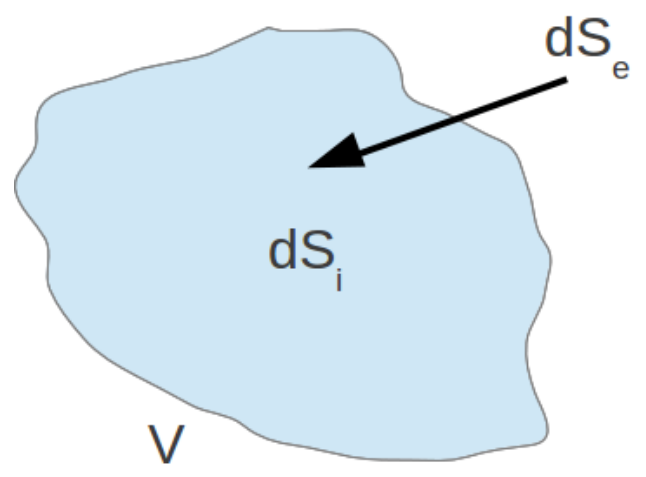

Figura 2.1: Variação de Entropia. A figura mostra onde e como atuam as diferentes contribuições para a variação de entropia, $d S$, no volume $V$. A variação de entropia interna $d S_{i}$ que ocorre como consequência dos fenomenos irreversíveis no interior de $V$. Já a variação de entropia externa, $d S_{e}$, atua no sistema através dos fluxos, isto é, sua contribuição depende do fluxo de calor trocado entre o sistema e a vizinhança.

positiva definida, isto é:

$$
d S_{i} \geq 0
$$

Um processo é dito reversível, quando a variação de entropia interna é nula $\left(d S_{i}=0\right)$. Nestes casos, o estado final do sistema pode ser revertido, através de um processo ou uma série deles, ao seu estado inicial. Se para um processo a variação é não nula $\left(d S_{i}>0\right)$, então entropia é gerada sistema. Tais processos são ditos irreversíveis, pois é impossível reverter o sistema ao estado inicial sem que haja uma variação negativa da entropia interna, algo que violaria a segunda lei $[8,26]$. Em contrapartida, não há restrições para $d S_{e}$, podendo assumir qualquer valor positivo ou negativo, dependendo apenas da interação do sistema com a vizinhança. Desta forma, e como ilustrado pela figura 2.1, vemos que $d S_{e}$ altera a entropia do sistema através dos fluxos presentes no sistema contido em $V$.

Para sistemas fechados, onde apenas calor é trocado entre sistema e vizinhança, temos pelo teorema de Carnot-Clausius:

$$
d S_{e}=\frac{d Q}{T},
$$

onde $d Q$ é o calor fornecido para o sistema pela vizinhança a temperatura absoluta $T$.

Usando (2.22) e (2.23) encontramos:

$$
d S \geq \frac{d Q}{T},
$$

a segunda lei da termodinâmica.

Os conceitos apresentados nesta seção não estão escritos adequadamente para o trata- 
mento no contexto das equações de balanço. Portanto é necessário que as expressões (2.22) e (2.23) sejam reescritas em termos de variáveis localmente definidas (tais como $\rho$ e $p$ ). Para isso escrevemos:

$$
\begin{array}{r}
S=\int_{V} \rho s d V, \\
\frac{d S_{e}}{d t}=-\int_{\boldsymbol{\Omega}} \mathbf{J}_{s, t o t} . d \mathbf{\Omega} \\
\frac{d S_{i}}{d t}=\int_{V} \sigma_{S} d V,
\end{array}
$$

onde $s$ é a entropia por unidade de massa, $\mathbf{J}_{s, t o t}$ é o fluxo total de entropia por unidade de área por unidade de tempo e $\sigma_{S}$ é a fonte de entropia por unidade de volume por unidadede tempo.

A variação da entropia com relação ao tempo pode ser obtida usando as equações (2.26), (2.27), (2.28) e com o auxílio do teorema de Gauss:

$$
\int_{V}\left(\frac{\partial \rho s}{\partial t}+\nabla \cdot \mathbf{J}_{s, t o t}-\sigma_{S}\right) d V=0
$$

Na dedução acima foram consideradas válidas as propriedades dadas em (2.22) e (2.23) para partes infinitesimais do sistema e, portanto, de acordo com a descrição macroscópica de um sistema contínuo [24].

Segue de (2.29) e (2.23):

$$
\begin{array}{r}
\frac{\partial \rho s}{\partial t}=-\nabla \cdot \mathbf{J}_{s, t o t}+\sigma_{S}, \\
\sigma_{S} \geq 0 .
\end{array}
$$

As duas equações acima representam a formulação local da segunda lei da termodinâmica. Note que (2.30) é a equação de balanço para a densidade de entropia com o termo de fonte $\sigma_{S}$ obedecendo a inequação (2.31).

\subsubsection{A Fonte de Entropia}

Nesta seção nos dedicamos a obter expressões detalhadas para os termos de fonte de entropia , $\sigma_{S}$, e fluxo de entropia, $\mathbf{J}_{s, t o t}$, utilizando as várias equações de balanço obtidas 
nas seções anteriores.

É sabido da termodinâmica que a entropia por unidade de massa, s, é uma função de parâmetros que definem completamente o estado macroscópico do sistema. Pelo tratamento apresentado até aqui $s$ pode ser escrita como função da energia interna e do volume específico, $s(u, v)$. No equilíbrio a diferencial total de $s$ é dada pela primeira lei da termodinâmica [24, 27]:

$$
T d s=d u+p d v
$$

onde $p$ é a pressão de equilíbrio.

A primeira lei é definida no estado de equilíbrio, contudo é possível utilizá-la em um caso particular fora do equilíbrio e que será apresentado a seguir. Vamos considerar o sistema como um todo fora do equilíbrio, mas que sempre seja possível encontrar pequenas regiões em seu interior localmente em equilíbrio. Nesta região é possível definir $s$ e, portanto, utilizá-la na descrição do sistema, esta hipótese é conhecida como equilíbrio local. A hipótese só pode ser justificada (do ponto de vista macroscópico) somente pelas conclusões que dela são obtidas ou através de uma abordagem via teoria microscópica [24].

Considerando a hipótese de equilíbrio local, a variação da primeira lei com o tempo é:

$$
T \frac{d s}{d t}=\frac{d u}{d t}+p \frac{d v}{d t}
$$

Se forem substituídas em (2.33) as equações (2.21) para $d u / d t$ e (2.20) para $d q / d t$, obtemos:

$$
\rho \frac{d s}{d t}=-\nabla \cdot\left(\frac{\mathbf{J}_{q}}{T}\right)-\frac{1}{T^{2}} \mathbf{J}_{q} \cdot \nabla T-\frac{1}{T} \boldsymbol{\Pi}: \nabla \mathbf{v} .
$$

Segue do apêndice B o tensor de pressão escrito em função dos coeficientes de viscosidade, o que permite reescrever a equação (2.34) como:

$$
\rho \frac{d s}{d t}=-\boldsymbol{\nabla} \cdot\left(\frac{\mathbf{J}_{q}}{T}\right)-\frac{1}{T^{2}} \mathbf{J}_{q} \cdot \nabla T-\frac{1}{T} \stackrel{\circ}{\boldsymbol{\Pi}}:(\stackrel{\circ}{\nabla} \mathbf{v})^{s}-\frac{1}{T} \Pi \nabla . \mathbf{v} .
$$

Em comparação com (2.30) seque que as expressões para o fluxo e a fonte de entropia 
são, respectivamente, dadas por:

$$
\begin{array}{r}
\mathbf{J}_{s}=\frac{\mathbf{J}_{q}}{T} \\
\sigma_{S}=-\frac{1}{T^{2}} \mathbf{J}_{q} \cdot \nabla T-\frac{1}{T} \stackrel{\circ}{\boldsymbol{\Pi}}:(\stackrel{\circ}{\nabla} \mathbf{v})^{s}-\frac{1}{T} \Pi \nabla \cdot \mathbf{v} \geq 0 .
\end{array}
$$

Vemos que o fluxo de entropia é dado pelo o fluxo de calor $\mathbf{J}_{q}$ dividido pela temperatura absoluta $T$, também observamos que não há fluxos de massa e portanto um sistema fechado. A fonte de entropia apresenta contribuições de três distintos mecânismos. O primeiro termo é relacionado a condução de calor, o segundo devido a viscosidade de cisalhamento (shear viscosity) e o terceiro devido a viscosidade volumar (bulk viscosity).

A fonte de entropia apresenta em (2.37) apresenta estrutura bilinear, ou seja, é o produto de um fluxo $\mathbf{J}$ (fluxo de calor $\mathbf{J}_{q}$, fluxo de momento ou tensor de pressão viscoso $\Pi$, etc) por um gradiente de intensidade (gradientes de temperatura, pressão, velocidade, etc). Esta quantidades que multiplicam os termos de fluxo na expressão para a produção de entropia são chamadas de "forças termodinâmicas".

\subsection{Positividade dos Coeficientes das Equações Fenomenológicas}

É conhecido empiricamente um grande número de fenômenos irreversíveis cujos fluxos são descritos por equações lineares. Tais equações recebem o nome de equações fenomenológicas de transporte por serem inseridas através de uma teoria fenomelógica e descreverem certos fenômenos de transporte. Podemos citar como exemplo a lei de Fourier para a condução do calor e a lei de Fick para a difusão. Estas equações também incluem os efeitos chamados de fenômenos cruzados, como por exemplo o efeito Soret [24].

Na sequência abaixos são escritas algumas destas equações:

$$
\begin{aligned}
& \mathbf{J}_{q}^{\prime}=-\kappa \boldsymbol{\nabla} T \\
& \stackrel{\circ}{\boldsymbol{\Pi}}=-\eta \stackrel{\circ}{\nabla} \mathbf{v} \\
& \Pi=-\eta_{v} \boldsymbol{\nabla} \cdot \mathbf{v}
\end{aligned}
$$

onde $\kappa$ é a condutividade térmica, $\eta$ a viscosidade e $\eta_{v}$ a viscosidade volumar, sendo todos 
escalares.

As equações fenomenológicas escritas aqui podem ser obtidas através das relações lineares de Onsager [25]. Tais equação são lineares e determinam a maneira mais simples de se escrever o transporte irreversível, preservando a positividade dos coeficientes.

Para um fluido livre de reações químicas, potenciais químicos $\mu_{i}$ nulos e na ausência de campos eletromagnéticos a expressão geral para a fonte de entropia é encontrada ao substituirmos as equações fenomenológicas citadas em (2.37):

$$
\sigma_{S}=\kappa \frac{(\boldsymbol{\nabla} T)^{2}}{T^{2}}+\frac{\eta}{T}(\boldsymbol{\nabla} \mathbf{v})^{2}+\frac{\eta_{v}}{T}(\boldsymbol{\nabla} \cdot \mathbf{v})^{2}
$$

Podemos observar que os únicos termos passíveis de serem negativos são apresentados ao quadrado, anulando a possibilidade da produção de entropia conter termos negativos. Como a condição de positividade da produção de entropia pode ser feita termo à termo, uma vez que os fenômenos de transporte podem existir independente uns dos outros, fica portanto mostrada a positividade do termo de produção de entropia para as equações fenomelógicas lineares.

\subsubsection{Fluidos na Presença de um Campo Elétrico}

Durante todo o capítulo nos atemos apenas ao tratamento de forças externas conservativas. Vamos agora apresentar uma breve discussão para um fluido na presença de um campo elétrico. O tratamento para estes tipos de forças é bastante semelhante ao caso de forças conservativas. Para o caso de um fluido de única componente, a principal diferença se encontra na determinação da energia total que deve contar a energia presente no campo eletromagnético.

Conhecendo a estrutura bilinear da fonte de entropia, podemos escrever para um fluido de única componente na presença de um campo elétrico $\mathbf{E}$ e na ausência de gradientes de temperatura e velocidade a seguinte relação (para maiores detalhes consultar [24] "NonEquilibrium Thermodynamics", Cap. XIII, publicado por Groot e Mazur):

$$
\sigma_{S}=z_{e} \frac{\mathbf{J} . \mathbf{E}}{T}
$$

onde $\mathbf{J}$, o fluxo conjugado, é a densidade de corrente e $z_{e}$ é a carga do elétron por unidade 
de massa.

As relações de Onsager [25] determinam que a forma mais simples de satizfazer a segunda lei da termodinâmica (a positividade da produção entropia) é uma relação linear do tipo:

$$
\mathbf{J}=\sigma \mathbf{E}
$$

onde $\mathbf{E}$ é a forção termodinâmica generalizada e $\sigma$ um coeficiente.

Esta equação é a conhecida Lei de Ohm [28], sendo $\sigma$ a condutividade elétrica e que podemos dizer ser demonstrada através da termodinâmica de não-equilíbrio. Substituindo (2.44) em (2.43), encontramos que a produção de entropia da seguinte forma:

$$
\sigma_{S}=\frac{\sigma z_{e}|\mathbf{E}|^{2}}{T}
$$

Dado que o único termo com possibilidade negativa está elevado ao quadrado na equação acima fica, portanto, provada a positividade da condutividade elétrica $\sigma$. 
Capítulo 3

\section{Teoria Cinética}

\subsection{Introdução}

Muitos autores acreditam que a teoria cinética moderna tenha se iniciado a partir das considerações efetuadas por D. Bernoulli [29], ao calcular cineticamente a pressão de um gás em equilíbrio termodinâmico $[9,11]$. O trabalho de Bernoulli foi tão radical para época que seus estudos foram continuados apenas por Clausius cerca de cem anos depois. Além do conceito de entropia, Clausius também introduziu a noção de livre-caminho médio e o modelo simplificado de gás ideal. Posteriormente, Maxwell introduziu o conceito de função de distribuição de velocidades e, entre outros resultados, mostrou que a lei de Boyle poderia ser calculada de primeiros princípios [30]. Em 1872, Boltzmann obteve a equação de evolução que leva seu nome, a partir da qual podemos dizer que a base conceitual da teoria cinética clássica foi finalmente estabelecida de forma mais completa e rigorosa, embora um amplo debate tenha ocorrido a respeito das hipóteses subjacentes ao chamado teorema $\mathrm{H}[35]$.

A descrição estatística de um gás proposta por Maxwell, através da função distribuição de velocidades, $f(\mathbf{v})$, ou simplesmente função distribuição, simplifica bastante a descrição do gás como um sistema de muitas partículas [7, 9, 10]. De fato, sabemos ser impossível o conhecimento detalhado de todas as condições iniciais das partículas de um gás, mesmo levando-se em consideração as modernas técnicas computacionais [8]. Uma importante característica notada por Maxwell na natureza estatística de uma gás é seu caráter irreversível e sua tendência de evoluir para um estado de equilíbrio termodinâmico, como posteriormente demonstrado por Boltzmann através do teorema $\mathrm{H}$.

A descrição irreversível macroscópica de um fluido, líquido ou gás, pode ser facilmente 
entendida através das chamadas equações de transporte (ver capítulo 2). Consideremos, por exemplo, a equação de Fourier para a condução do calor: $\mathbf{J}_{q}=-\kappa \boldsymbol{\nabla} T$, onde $\mathbf{J}_{q}$ é ligado ao movimento das partículas e $T$ proprocional a $\bar{v}^{2}$. Invertendo a direção das partículas vemos que a direção de $\mathbf{J}_{q}$ se inverte em ambos os casos, contudo $T$ não troca de sinal. Isto significa que a lei de Fourier é invariante por inversão temporal [8]. Esta constatação está contida no Teorema H através da sua ligação com a entropia.

Neste capítulo iremos apresentar um resumo da teoria cinética, bem como obter a equação de Boltzmann e seu termo colisional, além de calcularmos os coeficientes de transporte das equação fenomenológicas.

\subsection{Função de Distribuição de Velocidades}

Um importante conceito em teoria cinética é a função de distribuição de velocidades ou simplesmente função de distribuição, $f(\mathbf{r}, \mathbf{v}, t)[8,11]$. No estado de equilíbrio termodinâmico a função depende apenas da velocidade, isto é, $f=f(\mathbf{v})$. A partir da função de distribuição é possível determinar o estado do gás completamente, calcular os propriedades médias macroscópicas e os coeficientes de transporte das equações fenomenológicas apresentadas no capítulo 2. Este conceito foi proposto pela primeira vez por Maxwell ao descrever estatisticamente o estado de um gás monoatômico em equilíbrio termodinâmico [30]. O tratamento estatístico proposto por Maxwell esta de acordo com a hipótese de caos molecular proposta por Clausius e Boltzmann [31], uma vez que a correlação entre as velocidades das partículas ocorre apenas no instante da colisão.

\subsubsection{A Derivação de Maxwell}

Em 1860, Maxwell publicou uma dedução extremamente simples da função de distribuição para gases monoatômicos em equilíbrio termodinâmico [30]. Neste modelo a probabilidade de se encontrar uma molécula no elemento de volume do espaço de fase $d^{3} v$ em um gás homogêneo, mantido em repouso e livre de forças externas pode ser escrita como [11, 32]:

$$
\begin{array}{r}
f(\mathbf{v}) d^{3} v=\text { é o número de partículas com velocidade no intervalo } v_{i}+d v_{i} \\
\text { no elemento de volume } d^{3} v \text { do espaço de fase em torno de } \mathbf{v} .
\end{array}
$$


Devido a isotropia do gás no equilíbrio a funcão distribuição deverá ser a mesma em qualquer direção do espaço, consequentemente a relação $f(\mathbf{v})=f(-\mathbf{v})$ é satifeita. Com isto é possível observar que $f(\mathbf{v})$ será função de do módulo da velocidade, $|\mathbf{v}|=v$, ou equivalentemente $v^{2}[33]$. Também devemos supor os eventos estatisticamente independentes, o que permite escrever a probabilidade de encontrar a partícula na posição $\mathbf{v}$ a partir de suas componentes $v_{x}, v_{y}$ e $v_{z}$ :

$$
f(v) d^{3} v=f\left(v_{x}^{2}\right) f\left(v_{y}^{2}\right) f\left(v_{z}^{2}\right) d v_{x} d v_{y} d v_{z}
$$

A única função matemática que satisfaz a expressão acima é exponencial, portanto:

$$
f(v)=C e^{-a v^{2}}
$$

dado $C$ e $a$ constantes.

O coeficiente $C$ pode ser determinado a partir da definição do número total de partículas $[12,34]:$

$$
\int_{\mathbf{r}} \int_{\mathbf{v}} f(v) d^{3} v d^{3} r=N
$$

onde as integrais sobre $\mathbf{v}$ se estendem de $-\infty$ até $\infty$. É importante notar que a definição do número total de partículas também define a função distribuição como a densidade de pontos no espaço de fase.

Aplicando (3.3) em (3.4) encontramos o seguinte resultado:

$$
C V \int_{-\infty}^{\infty} e^{-a v^{2}} d^{3} v=N \quad \rightarrow \quad C=n\left(\frac{a}{\pi}\right)^{3 / 2}
$$

onde $n=N / V$ é a densidade de partículas do sistema, sendo $V$ o volume que o gás ocupa.

Para calcular o coeficiente $a$ podemos utilizar a definição de energia cinética média e sua relação com a temperatura [12, 34]:

$$
\frac{3}{2} n k_{B} T=\frac{1}{2} n m \bar{v}^{2}=\frac{1}{2} m \int_{v} f v^{2} d^{3} v
$$

onde $k_{B}$ é a constante de Boltzmann. 
Substituindo (3.3) encontramos:

$$
\frac{3}{2} n k_{B} T=3\left(\frac{C m}{n 2 a}\right)\left(\frac{\pi}{a}\right)^{3 / 2}
$$

Pelas equações (3.5) e (3.7) as constantes $C$ e $a$ podem ser escritas como:

$$
\begin{array}{r}
C=n\left(\frac{m}{2 \pi k_{B} T}\right)^{3 / 2}, \\
a=\frac{m}{2 k_{B} T} .
\end{array}
$$

Com o resultados acima a função apresentada em (3.3) é finalmente escrita como:

$$
f_{e q}(v)=n\left(\frac{m}{2 \pi k_{B} T}\right)^{3 / 2} e^{-\frac{m v^{2}}{2 k_{B} T}}
$$

No equilíbrio térmico vemos que não há dependência de $n$ e $T$ com $\mathbf{r}$ ou $t$, uma vez que o gás é homogêneo e estacionário . Sob estas circunstâncias dizemos que a equação (3.10) é a função de distribuição de Maxwell para um gás em equilíbrio térmico [7, 12, 32].

\subsubsection{Forma Local da Distribuição de Maxwell}

Existem inúmeras situações de interesse físico onde o gás não se encontra em equilíbrio térmico ou em regime estacionário, como por exemplo na presença de uma força externa ou mesmo numa estrela (sistema autogravitacional). Portanto é necessário que a função de distribuição passe a incluir as variáveis $\mathbf{r}$ como de $t$. Para casos ligeiramente fora do equilíbrio podemos acrescentar diretamente as dependências no espaço e no tempo nas variáveis termodinâmicas $T$ e $n$. Isto só é possível devido a inevitável tendência para equilíbrio (evidênciada pelo teorema $\mathrm{H}$ ) da função distribuição. Desta forma podemos escrever (3.10) como:

$$
f(\mathbf{r}, \mathbf{v}, t)=n(\mathbf{r}, t)\left(\frac{m}{2 \pi k_{B} T(\mathbf{r}, t)}\right)^{3 / 2} e^{-\frac{m v^{2}}{2 k_{B} T(\mathbf{r}, t)}}
$$




\subsection{Equação de Boltzmann}

Como visto na seção anterior a função de distribuição pode variar no tempo e no espaço, assim é importante determinar a evolução da função de distribuição em relação a estas variáveis. Tal evolução é determinada pela chamada equação de Boltzmann, uma equação integro-diferencial. Foi a partir desta equação que Boltzmann deduziu, com base numa descrição microscópica, a segunda lei da termodinâmica através de seu famoso teorema $\mathrm{H}$ [35].

Para deduzir a equação de Boltzmann vamos considerar um gás monoatômico e diluído sob a influência de uma força externa $\mathbf{F}$, de modo que podemos escrever a variação da velocidade $\mathbf{v}$ como $\mathbf{v}+\mathbf{F} / m d t$, enquanto a posição $\mathbf{r}$ é dada por $\mathbf{r}+\mathbf{v} d t$. Considerando a condição de normalização (3.4), podemos escrever o número de partículas no instante $t$ em um elemento diferencial de volume $d^{3} r d^{3} v$ no espaço de fase como $f(\mathbf{r}, \mathbf{v}, t) d^{3} r d^{3} v$ e sua evolução após um intervalo de tempo $d t$ como $f(\mathbf{r}+\mathbf{v} d t, \mathbf{v}+\mathbf{F} / m d t, t) d^{3} r d^{3} v$.

Devemos agora incluir o termo que determina a variação de partículas no elemento de volume no espaço de fase. Tal variação pode ser escrita como $(\delta f / \delta t)_{c o l} d t$, onde $d t$ é assumido como sendo muito maior que o tempo de interação entra as partículas durante as colisões. Esta suposição sempre será feita quando for tratado qualquer intervalo relacionado com as colisões das partículas, fazendo-se desnecessária sua repetição em situações posteriores.

Finalmente, podemos escrever que o número de partículas em um determinado elemento de volume $d^{3} r d^{3} v$ após um intervalo de tempo $d t$ dado por:

$$
\left[f\left(\mathbf{r}+\mathbf{v} d t, \mathbf{v}+\frac{\mathbf{F}}{m} d t, t+d t\right)-f(\mathbf{r}, \mathbf{v}, t)\right] d^{3} v d^{3} r=C(f) d^{3} r d^{3} v d t
$$

Expandindo e e tomando o limite $d t$ tendendo a zero obtemos:

$$
\frac{\partial f}{\partial t}+\mathbf{v} \cdot \nabla f+\frac{\mathbf{F}}{m} \cdot \nabla_{v} f=C(f) .
$$

A equação acima é conhecida como equação de Boltzmann [7, 8, 12], onde o termo $(\delta f / \delta t)_{\text {col }}$ descreve o efeito das colisões (usualmente chamado de termo colisional), e como veremos, é representado pela integral de colisões. Trataremos aqui apenas colisões binárias, ou seja, o encontro entre duas partículas de cada vez, assim desprezando colisões ternárias 


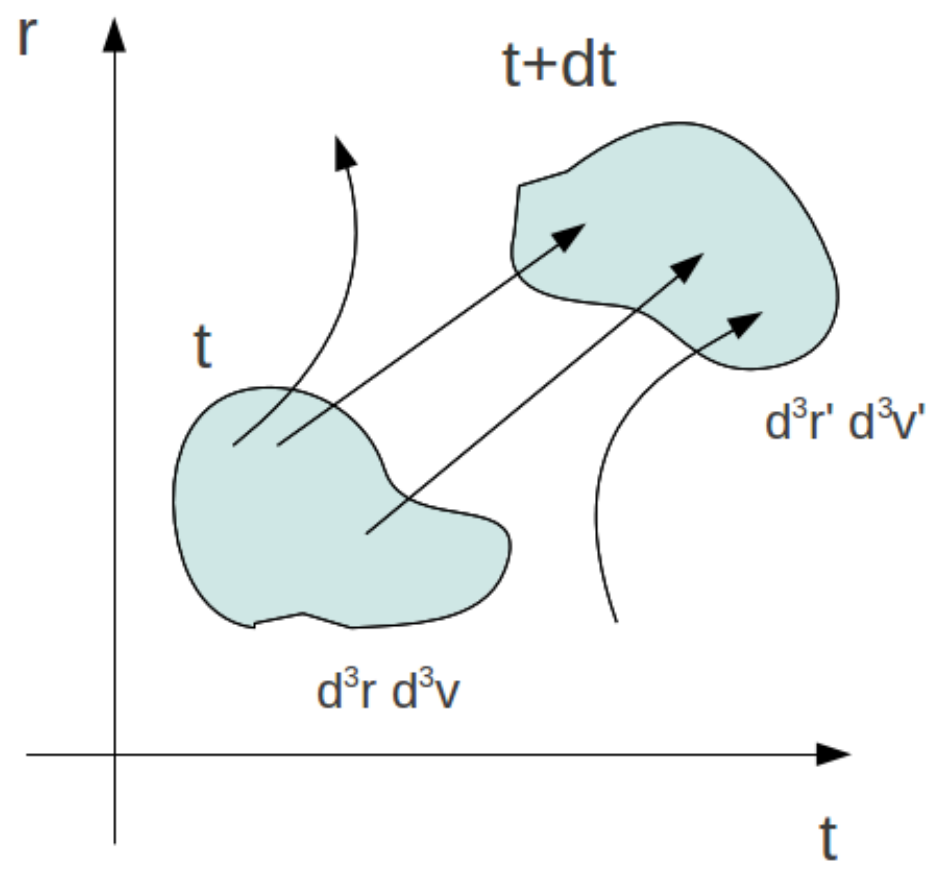

Figura 3.1: Efeitos das colisões na evolução do elemento de volume $d^{3} r d^{3} v$ do espaço de fase [12]. O efeito básico das colisões é variar o número líquido de partículas contido em um dado elemento de volume. Para o caso acolisional, ou seja, sem colisões, as setas que representam partículas entrando e saindo do elemento de volume devem ser eliminadas, uma vez que a densidade de fase é conservada (teorema de Liouville).

e quaternárias, uma vez que a probabilidade destas em um gás diluido é bastante pequena [35]. Também supomos colisões totalmente elásticas e que a interação entre as partículas ocorrer apenas no momento da colisão, desprezando eventuais correlação entre as partículas anteriores ou posteriores. Na ausência de colisões o gás é denominado não colisional. Neste caso otermo $C(f)$ da equação de Boltzmann é nulo, de modo que a equação (3.13) se reduz para:

$$
\frac{\partial f}{\partial t}+\mathbf{v} \cdot \nabla f+\frac{\mathbf{F}}{m} \cdot \nabla_{v} f=0 .
$$

A equação acima pode ser escrita de forma sucinta usando o operador $D / D t=\partial / \partial t+$ $\mathbf{v} \cdot \boldsymbol{\nabla}+\frac{\mathbf{F}}{m} \cdot \nabla_{v}:$

$$
\frac{D f}{D t}=0
$$

Esta condição representa a conservação de densidade de partículas no espaço de fase, em outras palavras, a densidade de partículas na vizinhança de um ponto que caminha no espaço de fase não se altera. Este resultado também é conhecido como Teorema de Liouville, reduzido a um espaço 6-D [12]. 


\subsubsection{Integral de Colisões de Boltzmann}

As colisões desempenham um importante papel na teoria cinética, são elas as responsáveis por conduzir o sistema ao equilíbrio e pelo transporte de quantidades como momento e energia, ambos processos irreversíveis. Elas aparecem na equação de Boltzmann através da integral de colisões, a qual iremos deduzir a seguir.

Vamos considerar partículas de dois tipos: as do tipo 1, representadas pela função distribuição $f_{1}\left(\mathbf{r}, \mathbf{v}_{1}, t\right)$ e as do tipo 2 pela função de distribuição $f_{2}\left(\mathbf{r}, \mathbf{v}_{2}, t\right)$. As partículas do tipo 1 podem deixar o elemento de volume que as contêm quando estas efetuam colisões com partículas do tipo 2. Da mesma forma, partículas do tipo 1 podem colidir com partículas do tipo 2 e entrar no elemento de volume $d^{3} r d^{3} v$. Vale resaltar que embora seja feita distinção entre as partículas, elas não precisam ser necessariamente de espécies diferentes, isto é feito em vista de distingui-las durante o processo de colisão, facilitando o entendimento da dedução da integral de colisões.

Definindo $\Delta N$ como a variação efetiva de partículas no elemento de volume $d^{3} r d^{3} v$ do espaço de fase durante o tempo $d t$, é possível escrever:

$$
\Delta N=C(f) d^{3} r d^{3} v d t
$$

É conveniente separar $\Delta N$ em duas partes, $\Delta N^{+}$para o ganho de partículas e $\Delta N^{-}$ para a perda de partículas. Para o cálculo de $\Delta N^{-}$vamos considerar partículas do tipo 1 situadas no elemento de volume $d^{3} r$ em $\mathbf{r}$ e $d^{3} v_{1}$ em $\mathbf{v}_{1}$ que colidem com partículas do tipo 2 no mesmo elemento de volume $d^{3} r$, mas cuja velocidade esta situada em $d^{3} v_{2}$ na coordenada $\mathbf{v}_{2}$. Sendo $f_{2}\left(\mathbf{r}, \mathbf{v}_{2}, t\right) d^{3} v_{2}$ o número de partículas do tipo 2 por unidade de volume com velocidades no elemento de volume $d^{3} v_{2}$ em $\mathbf{v}_{2}$, o fluxo de partículas incidentes pode ser expresso como [12]:

$$
\Gamma_{2}=f_{2}\left(\mathbf{r}, \mathbf{v}_{2}, t\right) d^{3} v_{2}\left|\mathbf{v}_{2}-\mathbf{v}_{1}\right|=f_{2}\left(\mathbf{r}, \mathbf{v}_{2}, t\right) v_{r e l} d^{3} v_{2}
$$

onde $v_{r e l}=\left|\mathbf{v}_{2}-\mathbf{v}_{1}\right|$ é a velocidade relativa entre as partículas que colidem.

Podemos considerar ainda que as partículas do tipo 2 se aproximam com o parâmetro de impacto situado entre $b$ e $b+d b$ e ângulo azimutal $\epsilon$ e $\epsilon+d \epsilon$. Ao passo do fluxo de 
partículas do tipo 2 pela área da seção de choque ser:

$$
\Gamma_{2} b d b d \epsilon d t=f_{2}(\mathbf{r}, \mathbf{v}, t) v_{r e l} b d b d \epsilon d t d^{3} v_{2}
$$

Esta expressão apenas nos fornece o número de partículas do tipo 2 com velocidade no elemento $d^{3} v_{2}$ na coordenada $\mathbf{v}_{2}$ e que estão situadas no elemento de volume de comprimento $v_{r e l} d t$ de seção de área $b d b d \epsilon$ como mostrado na Figura (3.2).

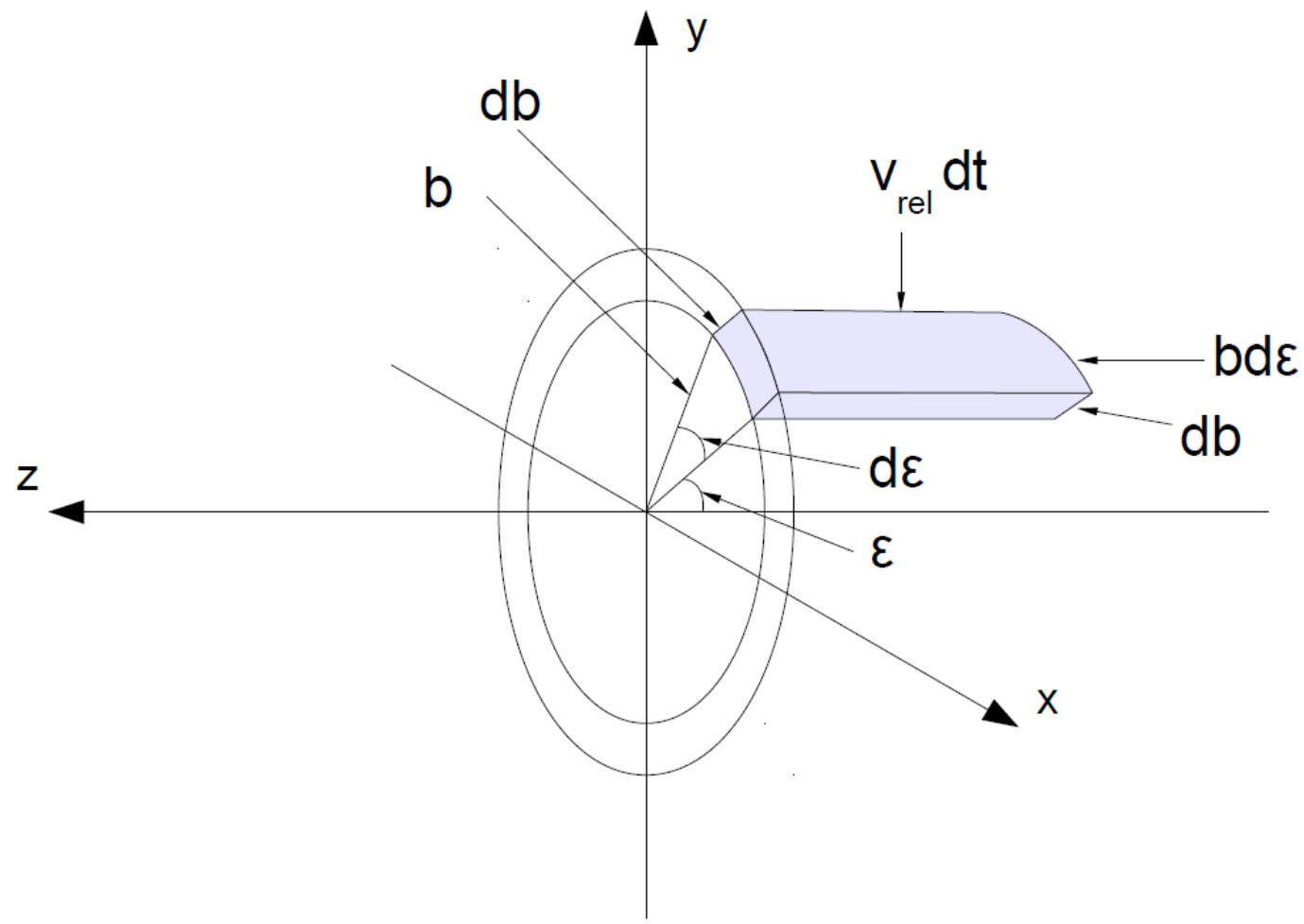

Figura 3.2: Cilindro de Colisão. O volume de comprimento $v_{r e l}$ dt e a seção de choque $b d b d \epsilon$, entre os intervalos do parâmetro de impacto $b$ e $b+d b$ e ângulo do plano de colisão $\epsilon$ e $\epsilon+d \epsilon$. No cálculo do termo colisional é suposto que as colisões são binárias e que o tempo dt é longo em comparação com o tempo de interação das partículas.

Para determinar o número de colisões entre as partículas 1 e 2 devemos multiplicar (3.18) por $f_{1}\left(\mathbf{r}, \mathbf{v}_{1}, t\right) d^{3} r d^{3} v_{1}$ :

$$
f_{1}\left(\mathbf{r}, \mathbf{v}_{1}, t\right) d^{3} r d^{3} v_{1} f_{2}\left(\mathbf{r}, \mathbf{v}_{2}, t\right) v_{r e l} b d b d \epsilon d t d^{3} v_{2}
$$

Na passagem anterior adotamos as colisões proporcionais ao produto $f_{1}\left(\mathbf{r}, \mathbf{v}_{1}, t\right) f_{2}\left(\mathbf{r}, \mathbf{v}_{2}, t\right)$, mais precisamente, a probabilidade de haver uma colisão é proporcional ao produto. Esta aproximação é conhecida como caos molecular.

O número total de partículas 1 em $d^{3} r$ defletidas para fora do elemento de volume $d^{3} v_{1}$ 
durante o tempo $d t$ é obtido integrando a expressão (3.19) sobre todos os valores possíveis de $b, \epsilon$ e $\mathbf{v}_{2}$ :

$$
\Delta N^{-}=\int_{v_{2}} \int_{b} \int_{\epsilon} f_{2}\left(\mathbf{r}, \mathbf{v}_{2}, t\right) f_{1}\left(\mathbf{r}, \mathbf{v}_{1}, t\right) v_{r e l} d^{3} r d^{3} v_{1} d^{3} v_{2} d t b d b d \epsilon
$$

onde a integral tripla em $\mathbf{v}_{2}$ é representada por uma única integral.

O ganho de partículas $\Delta N^{+}$é determinado de maneira similar ao $\Delta N^{-}$, apenas considerando colisões inversas. Suponhamos que partículas do tipo 1 no elemento de volume $d^{3} r$ na posição $\mathbf{r}$ e com velocidade $d^{3} v_{1}^{\prime}$ em $\mathbf{v}_{1}^{\prime}$ colidem com uma partículas do tipo 2 com velocidade $d^{3} v_{2}^{\prime}$ em $\mathbf{v}_{2}^{\prime}$ também na posição $\mathbf{r}$. Sendo $f_{2}(\mathbf{r}, \mathbf{v}, t) d^{3} v_{2}^{\prime}$ o número médio de partículas por unidade de volume no elemento de volume $d^{3} v_{2}^{\prime}$ em $\mathbf{v}_{2}^{\prime}$, podemos escrever o fluxo de partículas incidentes como:

$$
\Gamma_{2}^{\prime}=f_{2}\left(\mathbf{r}, \mathbf{v}_{2}, t\right) v_{r e l}^{\prime} d^{3} v_{2}^{\prime},
$$

onde $v_{r e l}^{\prime}=\left|\mathbf{v}_{2}^{\prime}-\mathbf{v}_{1}^{\prime}\right|$ é a velocidade relativa das colisões inversas.

Considerando agora que as partículas se aproximem com um parâmetro de impacto entre $b$ e $b+d b$ e ângulo azimutal $\epsilon$ e $\epsilon+d \epsilon$ no qual ocorre a colisão, o fluxo de partículas pela área da seção de choque é:

$$
\Gamma_{2}^{\prime} b d b d \epsilon d t=f_{2}\left(\mathbf{r}, \mathbf{v}^{\prime}, t\right) v_{r e l}^{\prime} b d b d \epsilon d t d^{3} v_{2}^{\prime}
$$

Multiplicando a equação (3.22) por $f_{1}\left(\mathbf{r}, \mathbf{v}_{1}^{\prime}, t\right) d^{3} r d^{3} v$ e integrando sob todos os valores possíveis de $b, \epsilon \mathrm{e}_{2}^{\prime}$, encontramos o número total de partículas do tipo 1 refletidas para dentro do elemento de volume $d^{3} v_{1}^{\prime}$ no intervalo de tempo $d t$ :

$$
\Delta N^{+}=\int_{v_{2}^{\prime}} \int_{b} \int_{\epsilon} f_{2}\left(\mathbf{r}, \mathbf{v}_{2}^{\prime}, t\right) f_{1}\left(\mathbf{r}, \mathbf{v}_{1}^{\prime}, t\right) v_{r e l}^{\prime} d^{3} r d^{3} v_{1}^{\prime} d^{3} v_{2}^{\prime} d t b d b d \epsilon
$$

Como o modulo da velocidade relativa não se altera durante as colisões inversas temos que $v_{r e l}^{\prime}=v_{r e l}=\left|\mathbf{v}_{2}-\mathbf{v}_{1}\right|$. Da teoria de jacobianos (consultar apêndice A) temos ainda que:

$$
d^{3} v_{1}^{\prime} d^{3} v_{2}^{\prime}=d^{3} v_{1} d^{3} v_{2}
$$

Aplicando as equações (3.20), (3.23) e (3.24) em (3.16) e ainda dividindo a expressão 
resultante por $d^{3} r d^{3} v d t$ :

$$
C(f)=\left(\frac{\Delta N^{+}-\Delta N^{-}}{d^{3} r d^{3} v d t}\right)=\int_{v_{2}} \int_{\theta}\left(f_{1}^{\prime} f_{2}^{\prime}-f_{1} f_{2}\right) v_{r e l} d \theta d^{3} v_{2}
$$

onde usamos

$$
\int_{\theta} d \theta=\int_{b} \int_{\epsilon} b d b d \epsilon
$$

e a notação

$$
\begin{array}{r}
f_{1}^{\prime}=f_{1}\left(\mathbf{r}, \mathbf{v}_{1}^{\prime}, t\right) \\
f_{2}^{\prime}=f\left(\mathbf{r}, \mathbf{v}_{2}^{\prime}, t\right) \\
f_{1}=f_{1}\left(\mathbf{r}, \mathbf{v}_{1}, t\right) \\
f_{2}=f_{2}\left(\mathbf{r}, \mathbf{v}_{2}, t\right) .
\end{array}
$$

Com o termo colisional a equação de Boltzmann pode ser escrita como:

$$
\frac{\partial f}{\partial t}+\mathbf{v} \cdot \nabla f+\frac{\mathbf{F}}{m} \cdot \nabla_{v} f=\int_{\theta} \int_{v_{2}}\left(f_{1}^{\prime} f_{2}^{\prime}-f_{1} f_{2}\right) v_{r e l} d \theta d^{3} v_{2}
$$

\subsubsection{Teorema H de Boltzmann}

Uma importante característica do termo colisional da equação de Boltzmann é conduzir a função de distribuição para o estado de equilíbrio de maneira irreversível [3, 12]. A característica da irreversíbilidade vem como consequência do tratamento estatístico do gás [8].

A fim de compreender melhor a irreversibilidade contida no termo colisional da equação de Boltzmann é definida a função $H(t)$. Considerando um gás formado de uma única espécie de partícula e descrito pela função de distribuição $f(\mathbf{r}, \mathbf{v}, t)$; a função $H(t)$ é escrita como:

$$
H(t)=\int_{r} \int_{v} f(\mathbf{r}, \mathbf{v}, t) \ln f(\mathbf{r}, \mathbf{v}, t) d^{3} r d^{3} v
$$

De modo que a função $H(t)$ se relaciona com a entropia $S$ da seguinte maneira $[3,8,12]$ :

$$
S=-k H(t)
$$

onde $k_{B}$ é a constante de Boltzmann. 
Através da equação (3.30) é claro que $H(t)$ deve satisfazer certas condições, uma vez que a entropia segue uma série de requisitos. O Teorema $\mathrm{H}$ de Boltzmann justamente mostra que tais requisitos são de fato respeitados pela função $H(t)$. Considerando $f(\mathbf{r}, \mathbf{v}, t)$ solução da equação de Boltzmann e seguindo a notação para as colisões diretas e inversas presente no quadro (3.27), podemos enunciar o teorema da seguinte maneira:

Teorema $H$ de Boltzmann - Se $f(\mathbf{r}, \mathbf{v}, t)$ é solução da equação de Boltzmann, isto é,

$$
\frac{\partial f}{\partial t}+\mathbf{v} \cdot \boldsymbol{\nabla} f+\frac{\mathbf{F}}{m} \cdot \nabla_{v} f=\int_{r} \int_{\theta} \int_{v_{2}}\left(f_{1}^{\prime} f_{2}^{\prime}-f_{1} f_{2}\right) v_{r e l} d \theta d^{3} r d^{3} v_{2},
$$

então

$$
\frac{\partial H(t)}{\partial t} \leq 0
$$

Prova do Teorema - Derivando a equação (3.29) em relação ao tempo:

$$
\frac{\partial H(t)}{\partial t}=\int_{r} \int_{v}(1+\ln f) \frac{\partial f}{\partial t} d^{3} r d^{3} v
$$

subtitituindo (3.31) em (3.33):

$$
\frac{\partial H(t)}{\partial t}=\int_{r} \int_{v_{1}} \int_{v_{2}}\left(1+\ln f_{1}\right)\left(\int_{\Omega}\left(f_{1}^{\prime} f_{2}^{\prime}-f_{1} f_{2}\right) v_{r e l} d \theta-\mathbf{v} \cdot \boldsymbol{\nabla} f_{1}-\frac{\mathbf{F}}{m} \cdot \nabla_{v} f_{1}\right) d^{3} r d^{3} v_{2} d^{3} v_{1} .
$$

Pelo teorema de Gauss, as integrais sobre o volume no espaço de fase envolvendo os termos $\boldsymbol{\nabla}$ e $\boldsymbol{\nabla}_{v}$ podem ser transformadas em integrais de superfície. Como o volume onde a função de distribuição $f$ assume valores não nulos no espaço de fase é finito e as integrais sobre $r$ e $v$ se estendem até o infinito, conluí-se que tais integrais são nulas.Portanto (3.34) pode ser reescrita como:

$$
\frac{\partial H(t)}{\partial t}=\int_{\theta} \int_{r} \int_{v_{1}} \int_{v_{2}}\left(1+\ln f_{1}\right)\left(f_{1}^{\prime} f_{2}^{\prime}-f_{1} f_{2}\right) v_{r e l} d \theta d \mathbf{r} d \mathbf{v}_{2} d \mathbf{v}_{1}
$$

Como as variáveis $\mathbf{v}$ e $\mathbf{v}_{2}^{\prime}$ são mudas podemos ainda reescrever (3.35) na seguinte forma:

$$
\frac{\partial H(t)}{\partial t}=\int_{\theta} \int_{r} \int_{v_{1}} \int_{v_{2}}\left(1+\ln f_{2}\right)\left(f_{2}^{\prime} f_{1}^{\prime}-f_{2} f_{1}\right) v_{r e l} d \theta d^{3} r d^{3} v_{2} d^{3} v_{1} .
$$


Somando (3.35) e (3.36):

$$
2 \frac{\partial H(t)}{\partial t}=\int_{\theta} \int_{r} \int_{v_{2}} \int_{v}\left[2+\ln \left(f_{1} f_{2}\right)\right]\left(f_{2}^{\prime} f_{1}^{\prime}-f_{2} f_{1}\right) v_{r e l} d \theta d^{3} r d^{3} v_{2} d^{3} v_{1} .
$$

É possível ainda trocar as velocidades $\mathbf{v}$ e $\mathbf{v}_{2}$, antes da colisão, pelas velocidades $\mathbf{v}^{\prime} \mathrm{e}$ $\mathbf{v}_{2}^{\prime}$, depois da colisão. Isso é possível devido a existência uma colisão inversa para cada colisão com a mesma seção de choque, logo:

$$
2 \frac{\partial H(t)}{\partial t}=\int_{\theta} \int_{r} \int_{v_{2}} \int_{v_{1}}\left[2+\ln \left(f_{1}^{\prime} f_{2}^{\prime}\right)\right]\left(f_{2}^{\prime} f_{1}^{\prime}-f_{2} f_{1}\right) v_{r e l} d \theta d^{3} r d^{3} v_{2} d^{3} v_{1}
$$

Combinando agora (3.37) e (3.38) encontra-se:

$$
\frac{\partial H}{\partial t}=\frac{1}{4} \int_{\Omega} \int_{r} \int_{v_{2}} \int_{v_{1}}\left[\ln \frac{f_{1} f_{2}}{f_{1}^{\prime} f_{2}^{\prime}}\right]\left(f_{2}^{\prime} f_{1}^{\prime}-f_{2} f_{1}\right) v_{r e l}, d \theta d^{3} r d^{3} v_{2} d^{3} v_{1} .
$$

Nota-se por esta expressão que se $f_{1}^{\prime} f_{2}^{\prime}>f_{1} f_{2}$ então $\ln \left(f_{1} f_{2} / f_{1}^{\prime} f_{2}^{\prime}\right)<0$ e, portanto, $(\partial H(t) / \partial t)<0$. Por outro lado, se $f_{1}^{\prime} f_{2}^{\prime}<f_{1} f_{2}$ então $\ln \left(f_{1} f_{2} / f_{1}^{\prime} f_{2}^{\prime}\right)>0$, mas $\left(f_{1} f_{2}-\right.$ $\left.f_{1}^{\prime} f_{2}^{\prime}\right)<0$, portanto $(\partial H(t) / \partial t)<0$. Quando $f_{1}^{\prime} f_{2}^{\prime}=f_{1} f_{2}$, ambos os fatores são zero $(\partial H(t)=0)$, o que corresponde ao estado de equilíbrio.

Este resultado prova o teorema $\mathrm{H}$, mostrando que se $f(\mathbf{r}, \mathbf{v}, t)$ satisfaz a equação de Boltzmann, a função $H(t)$ decresce monotonicamente até um valor limite, como mostrado na figura 3.3. É interessante notar que este resultado, juntamente com a relação entre a entropia e a função $H(t)$, define a entropia no contexto cinético. A definição cinética da entropia é uma consequência do comportamento estatístico das partículas do gás relacionado com a irreversibilidade imposta pela hipótese do caos molecular. Este valor limitante é alcançado apenas quando $f_{1}^{\prime} f_{2}^{\prime}=f_{1} f_{2}$, sendo esta a condição necessária para $\partial H(t) / \partial t=0$. Este é o chamado estado de equilíbrio e foi obtido pela primeira vez por Maxwell em 1867 [36].

Este resultado é facilmente observado na equação (3.31):

$$
\int_{\Omega} \int_{r} \int_{v_{2}}\left[f_{1}^{\prime} f_{2}^{\prime}-f_{1} f_{2}\right] g \sigma(\theta) d \theta d^{3} r d^{3} v_{2}=0
$$

Quando a integral de colisões é nula recupera-se a equação de Boltzmann não colisional, conforme apresentada na equação (3.14). 


\section{$\mathrm{H}(\mathrm{t})$}

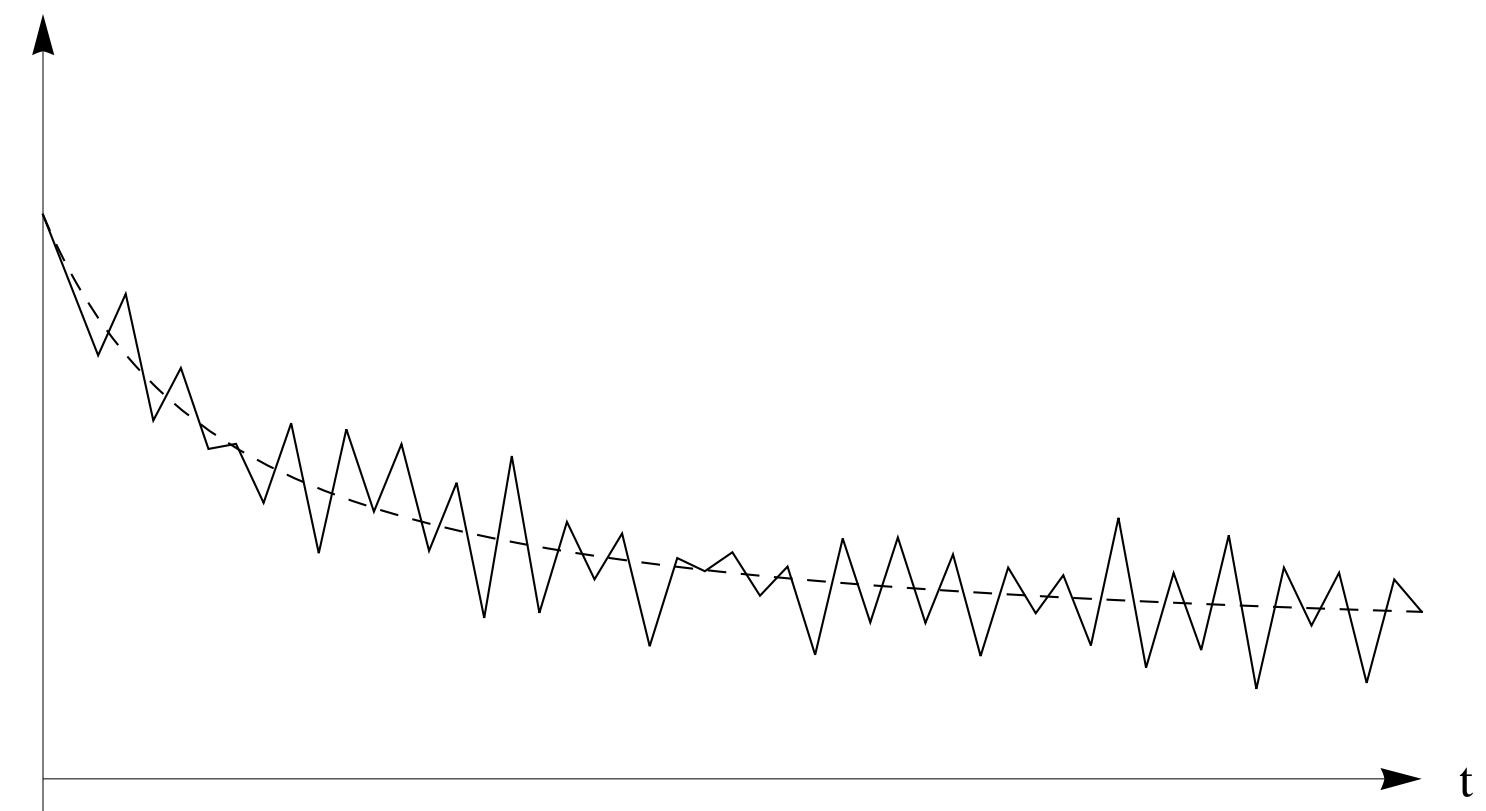

Figura 3.3: Teorema $\mathrm{H}$ e a tendência para o equilíbrio termodinâmico. A linha tracejada representa a solução prevista pela equação de Boltzmann para um gás inicialmente fora do equilíbrio. A linha sólida representa as flutuações de $H$ devido a hipótese probabilistica do caos molecular.

\subsubsection{Teorema H e a Distribuição Maxwelliana}

Como vimos, pelo teorema $\mathrm{H}$ o estado de equilíbrio é alcançado quando:

$$
f_{1}^{\prime} f_{2}^{\prime}=f_{1} f_{2} \text {. }
$$

Vamos mostrar agora que a partir da condição acima é possível encontrar a função de distribuição de velocidades para o equilíbrio, a distribuição de Maxwell. Podemos reescrever a (3.41) com ajuda da função logarítica ln da seguinte forma:

$$
\ln f_{1}^{\prime}+\ln f_{2}^{\prime}=\ln f_{1}+\ln f_{2} .
$$

Funções que obedecem relações como esta são chamadas de invariantes de soma e podem ser escritos como combinação de outros invariantes [7]. Em particular, podemos escrever $\ln f_{1}$, um invariante, da seguinte maneira [35]:

$$
\ln f=A+\mathbf{B} \cdot \mathbf{v}+D v^{2}
$$


onde omitimos o 1 uma vez que se trata de um índice auxíliar para a dedução do termo colisional.

Definindo as constantes $A, B$ e $D$ como:

$$
\begin{array}{r}
A=\operatorname{Ln} C-a u^{2}, \\
\mathbf{B}=2 a \mathbf{u}, \\
D=-a,
\end{array}
$$

podemos escrever a equação (3.43) como:

$$
f=C e^{-a(\mathbf{v}-\mathbf{u})^{2}}
$$

onde u é a velocidade média translacional do gás. As constantes $C$ e $a$ já foram calculadas na subseção 3.2.1, substituindo-as em (3.44) encontramos:

$$
f_{e q}=n\left(\frac{m}{2 \pi k_{B} T}\right)^{3 / 2} e^{-\frac{m v^{2}}{2 k_{B} T}}
$$

onde foi considerado $\mathbf{u}=0$.

Vemos portanto que a função distribuição de Maxwell pode ser obtida através da condição de equilíbrio determinada pelo teorema $\mathrm{H}$.

\subsubsection{Aproximação Tempo de Relaxação}

Na grande maioria dos casos a equação integro-diferencial de Boltzmann não é analiticamente solúvel. Isto acontece devido a integral de colisão ser dependente da própria função de distribuição que se deseja determinar (equação integro-diferencial). Uma maneira alternativa de abordar o problema (ainda que de forma aproximada) é propor modelos colisionais que substituam a integral, deixando a equação mais simples de ser resolvida. Neste sentido, um modelo bastante utilizado é conhecido por aproximação tempo de relaxação, originalmente proposto por Bhatnagar, Gross e Krook [14], sendo usualmente referido como modelo BGK (ver também [15]). Neste modelo é assumido que as colisões entre as partículas tendem a restaurar, de forma irreversível, o equilíbrio local através de um decaimento 
exponencial simples. Assim a integral de colisão é substituída pelo seguinte termo:

$$
C(f)=-\frac{f-f_{0}}{\tau}
$$

onde $f$ é função da posição, velocidade e do tempo, $f(\mathbf{r}, \mathbf{v}, t)$, e $\tau$ é o tempo de relaxação.

O tempo de relaxação $\tau$ representa uma espécie de tempo característico para o sistema atingir o equilíbrio $(t \gg \tau)$ [8]. Nota-se que atingido o equilíbrio, $f=f_{0}$, o efeito das colisões desaparece, tornando o estado equilíbrio independente do tempo como é fisicamente esperado.

Ao adotarmos o modelo tempo de relaxação, a equação de Boltzmann pode ser escrita como:

$$
\frac{\partial f}{\partial t}+\mathbf{v} \nabla f+\frac{\mathbf{F}}{m} \cdot \nabla_{v} f=-\frac{f-f_{0}}{\tau},
$$

onde $\mathbf{F} / m$ é a aceleração sentida pelas partículas do sistema devido a uma força externa.

Para compreendermos o significado físico do modelo BGK é interessante analisarmos um caso simples. Suponha um sistema compreendido como um plasma diluído, fracamente ionizado e na ausência de gradientes espaciais. Neste caso, a equação de Boltzmann na aproximação BGK toma a seguinte forma:

$$
\frac{\partial f}{\partial t}=-\frac{f-f_{0}}{\tau}
$$

Integrando a equação (3.48) encontramos a solução:

$$
f(\mathbf{v}, t)=f_{0}+\left(f(\mathbf{v}, 0)-f_{0}\right) e^{-t / \tau} .
$$

Vemos que o modelo BGK prevê um decaimento exponencial para o estado de equilíbrio, descrito por $f_{0}$ e governado pela escala de tempo $\tau$. O modelo colisional tempo de relaxação tem se revelado extremamente útil em inúmeras aplicações físicas, desde que a diferença $f-$ $f_{0}$ seja suficientemente pequena. Em tais casos as previsões do modelo fornecem resultados bem próximos aos obtidos resolvendo se a equação de Boltzmann sem qualquer tipo de simplificação ou mesmo numericamente. Contudo, por se tratar de uma simplificação, é de se esperar que a abordagem não prediga corretamente os resultados em várias situações, das quais podemos citar os casos envolvendo gradientes moderadamente intesos, ondas de 
choque, ondas sonoras ultra-sônicas, difusão na presença de reações químicas, efeito Nernst, processos de captura de elétrons, e, especialmente, em magnetoplasmas na presença de campos magnéticos intensos [3, 7, 8, 37, 38, 39].

\subsection{Cálculo dos Coeficientes de Transporte}

Desde sua descoberta por Boltzmann, a equação de evolução para a função de distribuição sempre se revelou de difícil solução. Ele próprio se concentrou nos calculos dos coeficientes de transporte mais simples. Algum avanço significativo veio apenas com Lorentz e sua proposta de aproximação que recebeu o nome de "gás de Lorentz"[40], um caso especial de um gás com partículas leves e pesadas.

Os trabalho subsequentes com relativo destaque, mas sem muito sucesso, foram os trabalhos de Hilbert [41], com sua expansão incompleta para a função de distribuição e Enskog [42], seguindo o método de expansão em potências para a velocidade peculiar iniciado por Boltzmann e que se mostrou intratável. Em 1916, Chapman usando a equação de transferência de Maxwell conseguiu obter expressões simples para o transporte em um gás com uma força geral que resolviam a equação de Boltzmann [43]. Posteriormente um trabalho de Enskog também obteve as mesmas equações de transporte utilizando o método de expansão agora conhecido como aproximação Chapman-Enskog [8].

Também podemos citar a contribuição creditada a Burnett [44], que ao invés de expandir a equação de Boltzmann em uma série de potências, utilizou polinômios de Sonine. Esta abordagem apresentou uma convergência mais rápida em relação as séries de Chapman-Enskog e coeficientes de transporte mais acurados. Outra contribuição significativa que podemos citar é a teoria de segunda ordem determinada por Grad [45].

Uma diferente forma na abordagem da equação de Boltzmann consiste na suposição de modelos colisionais que substituam a integral de colisões, transformando a equação integro-diferencial em uma simples equação diferencial. Neste contexto o modelo BGK [14] é amplamente difundido e embora simples, a aproximação linear produzida por ele é satisfatória na descrição de inúmeras situações. É através dessa abordagem que iremos calcular os coeficientes de transportes integrando diretamente a equação de Boltzmann. 


\subsubsection{Equação de Boltzmann Perturbada}

Como foi visto, o cálculo dos coeficientes de transporte via teoria cinética pode ser obtido através da solução da equação de transporte, a equação de Boltzmann [3]. Aqui iremos considerar um gás diluído em estado estacionário ou um plasma neutro, fracamente ionizado e ligeiramente fora do equilíbrio. Tais aproximações permitem escrever $f$, a função distribuição, que determina o estado do gás como uma simples perturbação do tipo $f=f_{0}+f_{1}, \operatorname{com}\left|f_{0}\right| \gg\left|f_{1}\right|$.

Vamos considerar também gradientes espaciais de $f_{1}$ sejam muito menores que para $f_{0}$, isto é, $\partial f_{0} / \partial \mathbf{x} \gg \partial f_{1} / \partial \mathbf{x}$, onde $\mathbf{x}$ denota a posição $\mathbf{r}$ ou a velocidade $\mathbf{v}$. Substituindo a função de distribuição na equação de Boltzmann (3.47) e considerando as aproximações acima, é fácil obter:

$$
f_{1}=-\tau \mathbf{v} \cdot \nabla f_{0}-\tau \frac{\mathbf{F}}{m} \cdot \nabla_{v} f_{0}
$$

onde $f_{0}$ é a função de distribuição do equilíbrio (3.11).

A expressão (3.50) fornece a solução para a perturbação da equação de Boltzmann linearizada obtida através do modelo BGK. A partir de agora, baseados nesta equação, e na distribuição de equilíbrio $f_{0}$, iremos calcular a condutividade térmica $(\kappa)$, a viscosidade $(\eta)$ para um gás e também a condutividade elétrica $(\sigma)$, esta última supondo que o sistema é formado por um plasma neutro diluído.

\subsubsection{Condutividade Térmica}

Quando existem diferenças de temperatura em um gás, um processo irrevesível no sentido de reconduzir o sistema ao estado equilíbrio termodinâmico ocorre. Este fenômeno é a condução de calor, ou seja, a transferência de calor através das ações das moléculas, que levam calor das regiões mais quentes para as mais frias do gás [9]. Este movimento determina a direção do fluxo como contrária a direção ao gradiente. Como é bem conhecido, na abordagem macroscópica o fluxo ou corrente de calor é descrito através de uma equação fenomenológica $\left(\mathbf{J}_{q}=-\kappa \boldsymbol{\nabla} T\right)$ como sendo o produto de um coeficiente (condutividade térmica) pelo gradiente de temperatura. No caso macroscópico não é possível determinálo teoricamente, fazendo-se necessário o uso de experimentos para obter sua dependência com relação nas quantidades termodinâmicas (temperatura, pressão, etc). No entanto, a abordagem propiciada pela teoria cinética permite o cálculo deste coeficiente, tratamento 
que apresentaremos a seguir.

Vamos agora calcular a condutividade térmica para um caso simples envolvendo um gás fora do equilíbrio descrito na aproximação BGK. Vamos supor também que o sistema é livre de campos externos, com pressão constante e coexiste com pequenos gradientes de tempertura. Nestas condições, o estado do gás pode ser descrito através da função de equilíbrio local (3.11) com a solução da equação de Boltzmann perturbada (3.50), se reduzindo para:

$$
f_{1}=-\tau\left[-\frac{5}{2}+\frac{m v^{2}}{2 k_{B} T(r)}\right] \frac{\mathbf{v} \cdot \nabla T(r)}{T(r)} f_{0}(\mathbf{r}, v)
$$

onde foi usado a seguinte relação $n \nabla T=-T \nabla n$, obtida a partir da equação de estado para os gases ideias $\left(p=n k_{B} T\right)[12]$.

A definição de fluxo de calor é dada por [12, 34]:

$$
\mathbf{J}_{q}=\frac{1}{2} m \int_{v} v^{2} \mathbf{v} f_{1}(\mathbf{r}, \mathbf{v}) d^{3} v
$$

Substituindo a função $f$ perturbada e lembrando que no equilíbrio não há gradientes de temperatura encontramos a seguinte equação para o fluxo de calor:

$$
\begin{array}{r}
\mathbf{J}_{q}=-\frac{1}{2} m \tau \int_{v} v^{2} \mathbf{v}\left[-\frac{5}{2}+\frac{m v^{2}}{2 k_{B} T(r)}\right] \times \\
\frac{\mathbf{v} \cdot \boldsymbol{\nabla} T(r)}{T(r)} f_{0}(\mathbf{r}, v) d^{3} v
\end{array}
$$

uma expressão que toma a seguinte forma em coordenadas esféricas:

$$
\mathbf{J}_{q}=-\frac{1}{2} m \tau \int_{0}^{\pi} \int_{0}^{2 \pi} \int_{0}^{\infty} v^{4} \mathbf{v}\left[-\frac{5}{2}+\frac{m v^{2}}{2 k_{B} T(r)}\right] \frac{\mathbf{v} \cdot \nabla T(r)}{T(r)} f_{0}(\mathbf{r}, v) \operatorname{sen} \theta d \theta d \phi d v
$$

Sabendo da seguinte relação [12]:

$$
\int_{0}^{\pi} \operatorname{sen} \theta d \theta \int_{0}^{2 \pi} \mathbf{v}[\mathbf{v} \cdot \nabla T(r)] d \phi=\frac{4 \pi}{3} v^{2} \nabla T(\mathbf{r})
$$

a equação (3.54) fica:

$$
\mathbf{J}_{q}=-\frac{2 \pi m \tau}{3} \frac{\nabla T(\mathbf{r})}{T(\mathbf{r})} \int_{0}^{\infty} v^{6}\left[-\frac{5}{2}+\frac{m v^{2}}{2 k_{B} T(\mathbf{r})}\right] f_{0} d v
$$


Efetuando a simples integral encontra-se o seguinte resultado:

$$
\mathbf{J}_{q}=-\frac{5 k_{B} p \tau}{2 m} \nabla T
$$

Comparando a equação (3.57) com a chamada Lei de Fourier (ver equação (2.38)) encontramos que a condutividade térmica é dada por:

$$
\kappa=\frac{5 k_{B} p \tau}{2 m}
$$

Vemos que a condutividade térmica é diretamente proporcional ao produto da pressão com o tempo médio de colisão $(\tau)$ e inversamente proporcional a massa das partículas do gás.

\subsubsection{Viscosidade}

Ordinarimente, a viscosidade aparece quando diferentes partes do gás estão se movendo com velocidades de cisalhamento distintas. Para facilitar, vamos considerar o caso mais simples, onde a direção da velocidades do gás do é igual em qualquer parte do mesmo, mas sua magnitude varia ponto a ponto. Se esta variação ocorre em uma dada direção preferencial, teremos um gradiente de velocidades. Sob estas condições encontra-se experimentalmente que a tensão sobre determinada camada no gás não é dada apenas pela pressão, ela também contem um termo tangencial (ou de cisalhamento). Esta componente tende a igualar as diferentes velocidadas das camadas, proporcionando a troca irreversível de momento cinético da camada de maior velocidade para a de menor velocidade [9]. Como já vimos, este fluxo é descrito por uma equação fenomenológica, onde a componente tangencial é proporcional ao gradiente de velocidade. Nosso objetivo agora será calcular este coeficiente de acordo com a teoria cinética (método BGK).

Consideremos um plasma fracamente ionizado e na ausência de campos externos e cuja velocidade média na direção $x$ é dada por $u_{x}(z)$. Nestas condições um gradiente de velocidades $\left(\partial u_{x}(z) / \partial z\right)$ surge na direção $z$, perpendicular a direção $x$ devido ao transporte de momento de maneira irreversível, como representado na figura a seguir: 


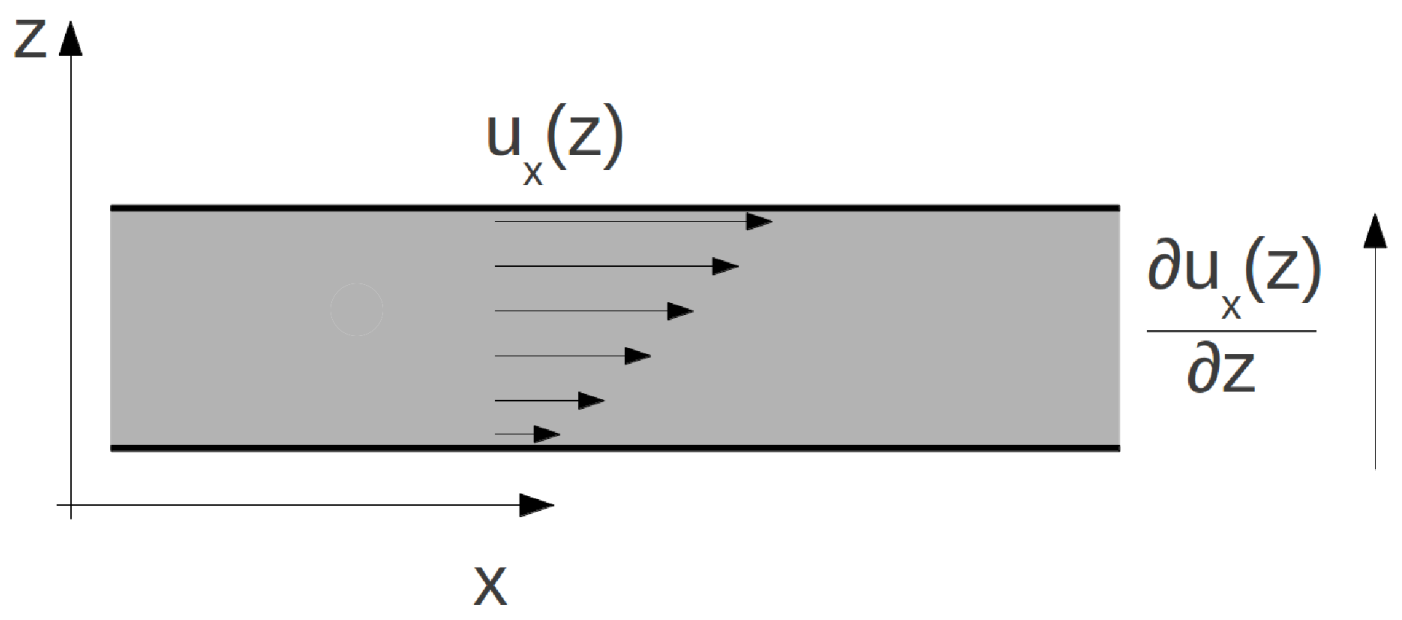

Figura 3.4: Representação de um gás dotado de um gradiente de velocidade. A figura representa um gás que escoa na direção $x$ e devido as condições de contorno apresenta um gradiente de velocidades na direção $z$, o que produz transporte de momento das regiões com maior momento para regiões de menor momento no gás. Este transporte perpendicular a direção da velocidade média do gás aparece nas componentes não diagonais no tensor de pressão e recebem o nome de tensão de cisalhamento.

Este resultado é expresso através do tensor de pressão $\mathrm{P}_{z x}$ e para o caso de pequenos gradientes pode ser expresso pela equação fenomenológica (2.39). Para calcularmos essa componente, precisamos da função de distribuição no equilíbrio, cuja expressão é dada por [32]:

Nestas circustâncias a função distribuição para o equilíbrio é dada como:

$$
f_{0}=n\left(\frac{m}{2 \pi k_{B} T}\right)^{\frac{3}{2}} e^{-\frac{1}{2} \frac{m}{k_{B} T}\left[\left(V_{x}-u_{x}(z)\right)^{2}+V_{y}^{2}+V_{z}^{2}\right]}
$$

onde $V_{i}$ com $i=x, y, z$ é a velocidade molecular.

Aplicando a função de distribuição (4.1) na equação de Boltzmann perturbada (3.50), lembrando a ausência de forças externas $(\mathbf{F}=0)$, encontramos:

$$
f_{1}=-v_{x} v_{z} \frac{m \tau}{k_{B} T} \frac{\partial u_{x}}{\partial z} f_{0}
$$

onde foi feito o uso da velocidade peculiar definida por $v_{i}=V_{i}-u_{i}, \forall i=x, y, z$.

A partir da definição do tensor de pressão [12, 34]:

$$
\mathrm{P}_{z x}=m \int v_{z} v_{x} f_{1} d^{3} v
$$


e utilizando as equações (3.60) e (3.59) em (3.61) obtemos:

$$
\mathrm{P}_{z x}=-\frac{m^{2} n \tau}{k_{B} T}\left(\frac{m}{2 \pi k_{B} T}\right)^{\frac{3}{2}} \int_{-\infty}^{\infty} \int_{-\infty}^{\infty} \int_{-\infty}^{\infty} v_{x}^{2} v_{z}^{2} e^{-\frac{1}{2} \frac{m}{k_{B} T}\left(v_{x}^{2}+v_{y}^{2}+v_{z}^{2}\right)} d v_{x} d v_{y} d v_{z}
$$

Por uma simples integração direta obtemos o seguinte resultado:

$$
\mathrm{P}_{z x}=-n k_{B} T \tau \frac{\partial u_{x}(z)}{\partial z}
$$

Comparando a equação acima com a equação fenomenológica para a o transporte de momemento (2.39) encontramos que o coeficiente de viscosidade assume a seguinte forma:

$$
\eta=n k_{B} T \tau
$$

\subsubsection{Condutividade Elétrica}

Os gases estudados até agora foram considerados não ionizados, isto é, de carga elétrica nula. Contudo um gás ligeiramente ionizado na presença de um campo elétrico $\mathbf{E}$ apresenta um comportamento de grande interesse em física de plasmas. As moléculas carregadas do gás se movem em resposta a presença do campo tendendo a anulação do mesmo. Os portadores de carga se aceleram devido a presença do campo, contudo são freados pelas colisões com outas partículas mais lentas. Em razão da direção preferencial induzida pelo campo elétrico, temos como resultado um fluxo de partículas carregadas. Este fluxo origina a corrente elétrica descrita experimentalmente através de uma equação fenomenológica linear (a lei de $\mathrm{Ohm}$ ), concebida como o força generalizada multiplicada por um coeficiente [25]. Passaremos agora ao da condutividade elétrica através da teoria cinética.

Vamos supor um plasma fracamente ionizado, homogêneo, não magnetizado em estado estacionário e equilíbrio térmico, colocado na presença de um campo elétrico orientado na direção $\hat{\mathbf{z}}$ de modo que é possível escrever $E \hat{\mathbf{z}}$. Nestas condições podemos usar a perturbação da equação de Boltzmann para encontrar a condutividade elétrica. Pelas as equações (3.50) e (3.11) encontramos:

$$
f_{1}=-\frac{e v E \tau}{k T} f_{0}
$$

onde $e$ é a carga do elétron. 
A definição de densidade de corrente é dada por [12, 34]:

$$
\mathbf{J}=-e \int_{v} \mathbf{v} f_{1} d^{3} v
$$

Sendo $f=f_{0}+f_{1}$ e $f_{0}$ a função de distribuição para o equilíbrio local (3.11), a integral acima pode ser escrita como:

$$
\mathbf{J}=\frac{e^{2} \mathbf{E} \tau}{k T} \int_{v} v^{2} f_{0} d^{3} v
$$

onde foi usado o fato $\mathbf{v} . \mathbf{E}=v E$, uma vez que a direção da velocidade dos elétrons é a mesma do campo externo aplicado no sitema.

Integrando (3.67) encontra-se:

$$
\mathbf{J}=\frac{n e^{2} \tau}{m} \mathbf{E}
$$

Comparando a equação (3.68) com a equação fenomenológica para a corrente elétrica (2.38) encontramos a condutividade elétrica como sendo:

$$
\sigma=\frac{n e^{2} \tau}{m}
$$

\subsubsection{Razão de Eucken}

A razão de Eucken $[20,46]$ se refera a uma quantidade adimensional medida experimentalmente entre a viscosidade $(\mu)$ e a condutividade térmica $(\kappa)$. Eucken sugeriu que a razão entre a $\kappa$ por $c_{v} \mu$, sendo $c_{v}$ o calor específico a volume constante, para gases poliatômicos é dada por:

$$
\Lambda \equiv \frac{\kappa m}{\eta k_{B}}
$$

Substituindo as equações da condutividade térmica a viscosidade, dadas respectivamente por (3.58) e (3.64), encontramos a razão como:

$$
\Lambda=\frac{5}{2}
$$

Pelo modelo BGK vemos que a raão de Eucken é uma constante igual a 2,5, contudo, dados experimentais mostram que a razão situa-se entre 2.6 e 1.3 [8, 46, 47]. 
Capítulo

\section{Modelos Colisionais Alternativos}

\subsection{Introdução}

Grande parte da dificuldade em se estudar fenômenos envolvendo colisões através da equação de Boltzmann se resume em solucioná-la [7]. Uma forma de contornar esta dificuldade é substituir a integral de colisões por um modelo colisional, transformando assim a equação integro-diferencial em uma equação diferencial. Contudo esta abordagem é limitada, uma vez que se trata de uma simplificação e, portanto, esperamos a existência de fenômenos de interesse físico que vão além dos limites pré-estabelecidos na aproximação adotada.

Neste capítulo propomos dois novos modelos colisionais. O primeiro modelo é apresentado como uma aproximação de segunda ordem do modelo conhecido como aproximação BGK [14]. O segundo modelo colisional é baseado em uma generalização do modelo BGK através de uma lei de potência. Para ilustrar uma aplicação dos modelos, calculamos para o modelo de segunda ordem os coeficientes de transporte das equações fenomenológicas apresentados na seção (3.4) e a chamada razão de Eucken. Para o modelo lei de potência, calculando a condutividade elétrica.

\subsection{Modelo Colisional com Termo de Segunda Ordem}

Como é bem conhecido o termo colisional adotado no modelo BGK, embora sendo de grande utilidade, é bastante limitado. Nesta seção discutiremos um novo modelo analítico, onde um termo adicional de segunda ordem é incluído, incorporando, naturalmente, efeitos não lineares na descrição dos fenômenos de transporte colisional.

Em analogia ao modelo linear, introduzimos um segundo tempo de relaxação, $\tau_{2}$, de- 
nominado de tempo de relaxação de segunda ordem. Este segundo tempo está relacionado com os efeitos não lineares que provocam desvios no decaimento exponencial presente no modelo linear.

Escrevemos o termo colisional com o termo de segunda ordem da seguinte maneira:

$$
\left(\frac{\delta f}{\delta t}\right)_{c o l}=-\frac{f-f_{0}}{\tau_{1}}-\frac{f_{0}}{\tau_{2}}\left(\frac{f-f_{0}}{f_{0}}\right)^{2}
$$

onde $\tau_{1}$ é o tempo de relaxação do modelo linear. No limite $\tau_{2} \gg \tau_{1}$ recupera-se o caso linear, ou seja, os efeitos não-lineares são desprezados desde o princípio.

A influência do termo de segunda ordem pode ser compreendida em um caso simples. Como, por exemplo, um gás (ou plasma neutro) diluído, na ausente de gradientes espaciais e campos externos. Sob tais condições, a equação de Boltzmann (3.13) toma a seguinte forma:

$$
\frac{\partial f}{\partial t}=-\frac{f-f_{0}}{\tau_{1}}-\frac{f_{0}}{\tau_{2}}\left(\frac{f-f_{0}}{f_{0}}\right)^{2} .
$$

Considerando uma mundança de variável $\mu=f / f_{0}-1$, encontramos a seguinte equação:

$$
\frac{\partial \mu}{\partial t}=-\frac{\mu}{\tau_{1}}-\frac{\mu^{2}}{\tau_{2}}
$$

A equação não homogênea e não linear acima é conhecida na literatura como equação de Riccati[48]. Para encontrarmos a solução, considere a substituição $\mu=-\frac{1}{\alpha} \frac{\dot{z}}{z}$, onde $\alpha$ é uma constante a ser determinada. Com isso a equação (4.3) é reescrita como:

$$
-\frac{1}{\alpha} \frac{\ddot{z}}{z}+\frac{1}{\alpha} \frac{\dot{z}^{2}}{z}-\frac{1}{\tau_{2} \alpha^{2}} \frac{\dot{z}^{2}}{z}-\frac{1}{\alpha \tau_{1}} \frac{\dot{z}}{z}=0 .
$$

A fim de eliminar os termos em $\dot{z}^{2}$, fixamos $\alpha=1 / \tau_{2}$, com (4.4) se reduzindo para:

$$
\frac{\ddot{z}}{\dot{z}}=-\frac{1}{\tau_{1}}
$$

e por uma integração direta encontramos a solução para $z(t)$ :

$$
z=-A \tau_{1} e^{-t / \tau_{1}}+B
$$

Utilizando a definição de $\mu(z, \dot{z})$ é fácil obter a seguinte solução geral para a função de 
distribuição:

$$
\frac{f-f_{0}}{f_{0}}=\frac{\tau_{2} A e^{-t / \tau}}{B-A \tau_{1} e^{-t / \tau_{1}}}
$$

Dada a condição inicial $t=0 \rightarrow f(\mathbf{v}, 0)$ encontramos $A$ em função de $B$ :

$$
A=\frac{B\left[f(\mathbf{v}, 0)-f_{0}\right]}{\tau_{2}\left[1+\frac{\tau_{1}}{\tau_{2}}\left(\frac{f(\mathbf{v}, 0)-f_{0}}{f_{0}}\right)\right]},
$$

e substituindo em (4.7), a solução da equação (4.2) pode ser escrita como:

$$
f(\mathbf{v}, t)=f_{0}+\frac{\left(f(\mathbf{v}, 0)-f_{0}\right) e^{-t / \tau_{1}}}{1+\frac{\tau_{1}}{\tau_{2}}\left(\frac{f(\mathbf{v}, 0)-f_{0}}{f_{0}}\right)\left(1-e^{-t / \tau_{1}}\right)} .
$$

Novamente podemos observar que no caso limite $\tau_{2} \gg \tau_{1}$ o decaimento exponencial do modelo linear é recuperado (ver equação (3.49)). Vemos também que a inserção do tempo característico de segunda ordem acelera o processo de decaimento para o equilíbrio, pois o denominador é maior do que a unidade para $t>0$.

É interessante ainda analisar o limite quando $\tau_{2}$ é grande o suficiente para que seja possível expandir o denominador da equação (4.9), mas não extremamente grande para evitar efeitos não-lineares. Tal limite é equivalente a dizer que o denominador na equação é pequeno e, portando, passível de expansão. Expandindo a equação (4.9) encontramos a seguinte expressão:

$$
f \approx f_{0}+\left(f(\mathbf{v}, 0)-f_{0}\right) e^{-t / \tau_{1}}\left(1-\frac{\tau_{1}}{\tau_{2}} f_{0}\left(\frac{f(\mathbf{v}, 0)-f_{0}}{f_{0}}\right)\right)
$$

Note que da equação acima podemos esperar correções da ordem $\tau_{1} / \tau_{2}$, quando utilizarmos o modelo colisional de segunda ordem para resolver a equação de Boltzmann. Em outras palavras, quando o gás estiver apenas ligeiramente deslocado do equilíbrio, esperamos que a solução geral seja dada pela soma da solução de primeira ordem mais um termo referente aos termos de segunda ordem.

Também é possível ver que as equações (4.9) e (4.10) decaem monotonicamente para um valor limite, o estado de equilíbrio caracterizado pela função de distribuição $f_{0}$. Este comportamento é esperado para modelos colisionais que satisfazem teorema $\mathrm{H}$, embora nesta dissertação não apresentaremos rigosmente a prova. 
A partir da equação (4.9) podemos fazer o seguinte gráfico que ilustra o decaimento para alguns valores da razão $\tau_{1} / \tau_{2}$ :

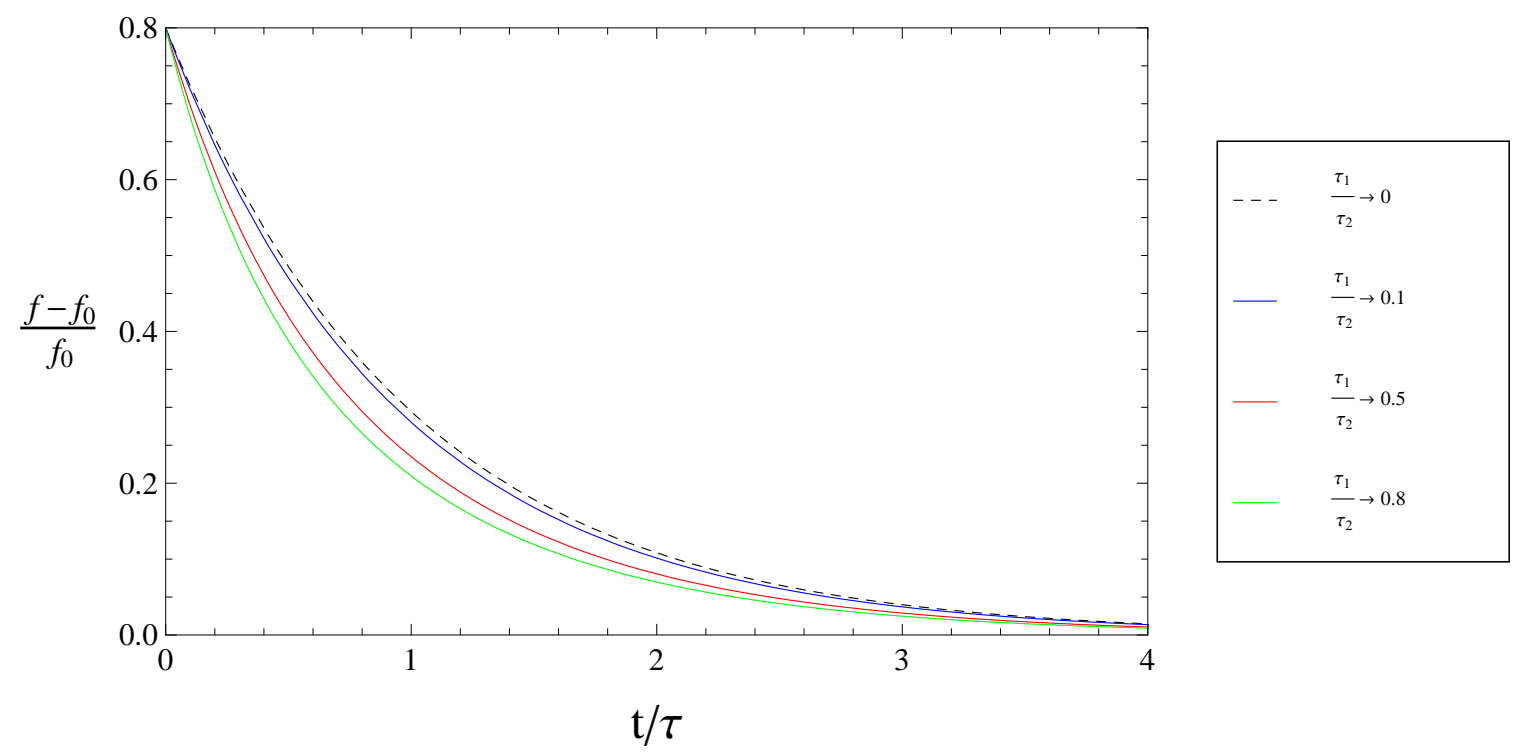

Figura 4.1: Decaimento para o equilíbrio na aproximação de segunda ordem. O gráfico mostra o decaimento em direção ao equílibrio para alguns valores da razão $\tau_{1} / \tau_{2}$ e o caso limite $\tau_{2} \gg \tau_{1}$ (representado por $\tau_{1} / \tau_{2}=0$ ), onde o decaimento exponencial previsto pelo modelo BGK é recuperado. Notamos ainda que o tempo necessário para atingir o equilíbrio termodinâmico através do modelo de segunda ordem é sempre menor que o caso exponencial.

Ainda foi assumido para traçar o gráfico da função que:

$$
\frac{f(\mathbf{v}, 0)-f_{0}}{f_{0}}=0.8
$$

Isso se deve a condição inicial e equilíbrio serem independentes do tempo, portanto, uma constante.

\subsection{Calculo dos Coeficientes de Transporte}

\subsubsection{Equação de Boltzmann Perturbada}

Tal como no capítulo 3, consideraremos agora um gás diluído, em regime estacionário e coexistindo com pequenos gradientes no espaço e no campo de velocidades. Sob tais circunstâncias, a equação de Boltzmann com o termo colisional de segunda ordem tem a forma:

$$
\mathbf{v} \cdot \nabla f+\frac{\mathbf{F}}{m} \cdot \nabla_{v} f=-\frac{f-f_{0}}{\tau_{1}}-\frac{f_{0}}{\tau_{2}}\left(\frac{f-f_{0}}{f_{0}}\right)^{2} .
$$


Sendo os gradientes em $\mathbf{r}$ e $\mathbf{v}$ pequenos, a perturbação induzida por eles também será pequena, sendo possível expandir $f$ em torno do equilíbrio dada por $f=f_{1}+f_{0}$. Neste caso a equação de Boltzmann (4.12) se reduz para:

$$
\frac{f_{1}}{\tau_{1}}+\frac{f_{1}^{2}}{\tau_{2} f_{0}}=-\mathbf{v} \cdot \nabla f_{0}-\frac{\mathbf{F}}{m} \cdot \nabla_{v} f_{0},
$$

onde foram considerados $\nabla f_{1} \ll \nabla f_{0}$ e $\nabla_{v} f_{1} \ll \nabla_{v} f_{0}$, ou seja, o gradiente espacial da perturbação é muito menor que o gradiente espacial do estado de equilíbrio.

Em termos de $f_{1}$, a equação (4.13) pode ser escrita como:

$$
f_{1}=-\frac{\tau_{2} f_{0}}{2 \tau_{1}}\left(1 \pm \sqrt{1-\frac{4 \tau_{1}^{2} \mathbf{v} \cdot \boldsymbol{\nabla} f_{0}}{\tau_{2} f_{0}}-4 \frac{\tau_{1}^{2} \mathbf{F} \cdot \nabla_{v} f_{0}}{m \tau_{2} f_{0}}}\right)
$$

Como supomos a perturbação pequena, podemos expandir o termo no interior da raíz. Impondo que a solução linear seja obtida no limite $\tau_{2} \gg \tau_{1}$ (ver discussão abaixo de (4.10)), encontramos a seguinte solução:

$$
\begin{aligned}
f_{1}= & -\tau_{1} \mathbf{v} \cdot \boldsymbol{\nabla} f_{0}-\tau_{1} \frac{\mathbf{F}}{m} \cdot \nabla_{v} f_{0}-\frac{\tau_{1}^{3}}{\tau_{2}} \frac{\left(\mathbf{v} \cdot \boldsymbol{\nabla} f_{0}\right)^{2}}{f_{0}} \\
& -\frac{\tau_{1}^{3}}{\tau_{2} f_{0}}\left(\frac{\mathbf{F}}{m} \cdot \nabla_{v} f_{0}\right)^{2}-2 \frac{\tau_{1}^{3}}{m \tau_{2} f_{0}}\left(\mathbf{v} \cdot \boldsymbol{\nabla} f_{0} \mathbf{F} \cdot \nabla_{v} f_{0}\right)-4 \frac{\tau_{1}^{5}}{\tau_{2}^{2}} \frac{\left(\mathbf{v} \cdot \boldsymbol{\nabla} f_{0}\right)^{3}}{f_{0}^{2}} \\
& -12 \frac{\tau_{1}^{5}}{\tau_{2}^{2}} \frac{\left(\mathbf{v} \cdot \boldsymbol{\nabla} f_{0}\right)^{2}\left(\mathbf{F} \cdot \boldsymbol{\nabla}_{v} f_{0}\right)}{m f_{0}^{2}}-12 \frac{\tau_{1}^{5}}{\tau_{2}^{2}} \frac{\left(\mathbf{v} \cdot \nabla_{v} f_{0}\right)\left(\mathbf{F} \cdot \nabla_{v} f_{0}\right)^{2}}{m^{2} f_{0}^{2}}-4 \frac{\tau_{1}^{5}}{\tau_{2}^{2}} \frac{\left(\mathbf{F} \cdot \boldsymbol{\nabla}_{v} f_{0}\right)^{3}}{m^{3} f_{0}^{2}}
\end{aligned}
$$

Note que os dois primeiros termos do lado direito na equação acima referida são a solução do modelo BGK (ver equação (3.50)). Os termos remanescentes são as contribuições de ordem superior que se anulam quando $\tau_{2}$ é muito grande $\left(\tau_{2} \gg \tau_{1}\right)$. Também podemos ver nesta equação a ocorrência de correções que dependem mutuamente dos gradientes da posição $\mathbf{r}$ e da velocidade v. Outro aspecto interessante da solução é o seguinte: de acordo com a discussão da seção (4.2), esperamos que para pequenas perturbações (portanto, com o sistema ligeiramente deslocado do equilíbrio) a solução possa ser escrita como a soma da solução linear (ver (3.50)) mais as correções de ordem superior, a mesma forma obtidida na equação acima.

A partir da solução (4.15), calcularemos os coeficientes generalizados, a saber: a con- 
dutividade térmica $(\kappa)$, a viscosidade $(\eta)$ e a condutividade elétrica $(\sigma)$.

\subsubsection{Condutividade Térmica}

Para calcular a condutividade térmica utilizando o modelo colisional de segunda ordem, adotaremos as mesmas hipóteses para o regime do gás apresentadas na seção (3.4.2). Sob tais condições podemos escrever a função de distribuição perturbada da seguinte forma:

$$
\begin{aligned}
f_{1}= & -\left(\left[-\frac{5}{2}+\frac{m v^{2}}{2 k_{B} T}\right] \frac{\tau_{1} \mathbf{v} \cdot \boldsymbol{\nabla} T}{T}+\frac{\tau_{1}}{\tau_{2}}\left[-\frac{5}{2}+\frac{m v^{2}}{2 k_{B} T}\right]^{2}\left(\frac{\tau_{1} \mathbf{v} \cdot \boldsymbol{\nabla} T}{T}\right)^{2}\right. \\
& \left.-4 \frac{\tau_{1}^{3}}{\tau_{2}^{2}}\left[-\frac{5}{2}+\frac{m v^{2}}{2 k_{B} T}\right]^{3}\left(\frac{\tau_{1} \mathbf{v} \cdot \boldsymbol{\nabla} T}{T}\right)^{3}\right) f_{0}
\end{aligned}
$$

Substituindo a solução acima na definição de fluxo de calor (3.52) obtemos:

$$
\begin{aligned}
\mathbf{J}_{q}= & -\frac{1}{2} m \int_{v} \mathbf{v} v^{2} \tau_{1}\left[-\frac{5}{2}+\frac{m v^{2}}{2 k_{B} T}\right] \frac{\mathbf{v} \cdot \boldsymbol{\nabla} T}{T} f_{0} d^{3} v \\
& -\frac{1}{2} m \int_{v} \mathbf{v} v^{2} \frac{\tau_{1}^{3}}{\tau_{2}}\left[-\frac{5}{2}+\frac{m v^{2}}{2 k_{B} T}\right]^{2}\left(\frac{\mathbf{v} \cdot \boldsymbol{\nabla} T}{T}\right)^{2} f_{0} d^{3} v \\
& -\frac{1}{2} m \int_{v} \mathbf{v} v^{2} \frac{\tau_{1}^{5}}{\tau_{2}^{2}}\left[-\frac{5}{2}+\frac{m v^{2}}{2 k_{B} T}\right]^{3}\left(\frac{\mathbf{v} \cdot \boldsymbol{\nabla} T}{T}\right)^{3} f_{0} d^{3} v .
\end{aligned}
$$

A primeira integral já foi resolvida (ver equação (3.57)), nos resta então calcular as outras duas. A partir da seguinte relação de ortogonalidade [12]:

$$
\int_{\theta} \int_{\phi} v_{i} v_{j} v_{k} \operatorname{sen} \theta d \theta d \phi=0
$$

onde $i, j, k=x, y, z$ são as componentes do vetor velocidade $\mathbf{v}$ em coordenadas esféricas é fácil verificar que a segunda integral é nula. Substituindo $f_{0}$ (definido em (3.11)), podemos reescrever a terceira integral na seguinte forma:

$$
-\frac{8 \pi m n}{3}\left(\frac{m}{2 \pi k T}\right)^{3 / 2} \frac{\tau_{1}^{3}}{\tau_{2}} \int_{0}^{2 \pi} \int_{0}^{\pi} \int_{0}^{\infty} v^{4} \mathbf{v}\left(-\frac{5}{2}+\frac{m v^{2}}{2 k T}\right)^{3}\left(\frac{\tau_{1} \mathbf{v} \cdot \nabla T}{T}\right)^{3} \operatorname{sen} \theta d v d \theta d \phi
$$

Efetuando a integral acima encontramos o resultado

$$
-184,8 \frac{5 k p \tau_{1}}{2 m} \frac{\tau_{1}^{2}}{\tau_{2}^{2}}\left(\frac{\tau_{1}}{\tau_{2}} \frac{l|\nabla T|}{T}\right)^{2} \nabla T=-184,8 \kappa \frac{\tau_{1}^{2}}{\tau_{2}^{2}}\left(\frac{l|\nabla T|}{T}\right)^{2} \nabla T .
$$


sendo $\kappa$ a condutividade térmica obtida através do modelo BGK (ver equação (3.58)). A partir deste resultado obtemos a expressão final para o fluxo de calor, no contexto do modelo colisional de segunda ordem:

$$
\mathbf{J}_{q}=-\kappa\left(1+184,8\left(\frac{\tau_{1}}{\tau_{2}} \frac{l|\nabla T|}{T}\right)^{2}\right) \nabla T,
$$

onde o caso linear é recuperado no limite $\tau_{2} \gg \tau_{1}$.

Comparando a equação (4.21) com a equação fenomenológica para a condução do calor (2.38) e com a equação da condutividade térmica no caso linear (3.58), obtemos a seguinte expressão para a condutividade térmica prevista pelo modelo BGK estendido:

$$
\kappa^{(2)}=\kappa\left(1+184,8\left(\frac{\tau_{1}}{\tau_{2}} \frac{l|\nabla T|}{T}\right)^{2}\right) .
$$

Como seria fisicamente esperado, a correção obtida acrescenta um termo proporcional ao módulo da força termodinâmica, $|\nabla T|$, de ordem $\tau_{2} / \tau_{1}$. Desta forma, vemos que a perturbação influencia diretamente o fenômeno de transporte alterando o valor da condutividade térmica.

É interessante notar que a participação do gradiente de temperatura na correção inclue também um novo comprimento característico ao problema relacionado aos fenômenos colisionais não lineares atuantes. Definimos este comprimento característico da seguinte forma:

$$
\frac{1}{L_{T}}=\frac{|\nabla T|}{T}
$$

onde o índice $T$ faz referência ao gradiente de temperatura.

Substituindo esta definição na equação (4.22) encontramos a seguinte expressão para a condutividade térmica de segunda ordem:

$$
\kappa^{(2)}=\kappa\left(1+184,8\left(\frac{\tau_{1}}{\tau_{2}} \frac{l}{L_{T}}\right)^{2}\right) .
$$

A razão $l / L_{T}$ é denominada "número de Knudsen", $K_{n}$, e pode ser entendido como uma influência das dimensões macroscópicas do problema sobre as dimensões características microscópicas do sistema [8], isto é, caso a razão não seja suficientemente pequena as dimensões características devem influenciar na evolução do sistema e, portante, devem 
aparecer naturalmente na descrição.

Sendo $L_{T}$ o comprimento característico associado aos fenômenos não-lineares, podemos supor um relação deste com o tempo característico dos fenômenos não-lineares, $\tau_{2}$. Em analogia ao tempo de relação de primeira ordem podemos escrever:

$$
\tau_{2}=\frac{L_{T}}{\bar{v}}
$$

onde $\bar{v}$ é a velocidade média. Assim podemos dizer que a equação (4.25) define numericamente o tempo de relaxação de segunda ordem.

Substituindo a expressão para $\tau_{2}$ em $(4.22)$ e também a relação para o comprimento característico $L_{T}$ (4.23), encontramos a condutividade térmica em função do número de Knudsen:

$$
\kappa^{(2)}=\kappa\left(1+184,8 K_{n_{T}}^{4}\right)
$$

onde o subíndice $T$ indica o número de Knudsen relativo ao comprimento característico do gradiente de temperatura.

Vemos que correções introduzidas pelo modelo colisional de segunda ordem são completamente determinadas pelo número de Knudsen, $K_{n_{T}}$. Este é um resultado interessante, pois se espera que correções de ordem superior nos fluxos lineares sejam expressas por potências do número de Knudsen [3, 8].

\subsubsection{Viscosidade}

Nesta seção, consideraremos as mesmas suposições adotadas anteriormente na seção (3.4.3) para a determinação da viscosidade. Portanto, considerando um gás diluído, não ionizado e dotado de uma velocidade média na direção $x$ dada por $u_{x}(z)$. Como a velocidade média depende da coordenada $z$ um gradiente é induzido, $\partial u_{x}(z) / \partial z$. Sendo a função $f_{0}$ dada por (3.59), temos a seguinte solução da equação de Boltzmann para a perturbação (em segunda ordem):

$$
\begin{aligned}
f_{1}= & -v_{x} v_{z} \frac{m \tau_{1}}{k_{B} T} \frac{\partial u_{x}}{\partial z} f_{0}-\frac{\tau_{1}}{\tau_{2}}\left(v_{x} v_{z} \frac{m \tau_{1}}{k_{B} T} \frac{\partial u_{x}}{\partial z}\right)^{2} f_{0} \\
& -\frac{\tau_{1}^{2}}{\tau_{2}^{2}}\left(v_{x} v_{z} \frac{m \tau_{1}}{k_{B} T} \frac{\partial u_{x}}{\partial z}\right)^{3} f_{0},
\end{aligned}
$$


de tal forma que para $\tau_{2} \gg \tau_{1}$, a solução é a mesma fornecidade pela equação (3.60).

Substituindo a expressão acima na definição do tensor de pressão (3.61), encontramos a seguinte expressão para o componente de pressão $\mathrm{P}_{z x}$ :

$$
\begin{aligned}
\mathrm{P}_{z x}= & -\frac{m^{2} \tau_{1}}{k_{B} T} \frac{\partial u_{x}}{\partial z} \int v_{x}^{2} v_{y}^{2} f_{0} d^{3} v-m \frac{\tau_{1}}{\tau_{2}}\left(\frac{m \tau_{1}}{k_{B} T} \frac{\partial u_{x}}{\partial z}\right)^{2} \int v_{x}^{3} v_{z}^{3} f_{0} d^{3} v \\
& -m \frac{\tau_{1}^{2}}{\tau_{2}^{2}}\left(\frac{m \tau_{1}}{k_{B} T} \frac{\partial u_{x}}{\partial z}\right)^{3} \int v_{x}^{4} v_{z}^{4} f_{0} d^{3} v
\end{aligned}
$$

onde as integrais triplas sob todo o espaço de fase foram condensadas em uma única integral.

Para determinar a viscosidade neste contexto, precisamos efetuar as integrais acima. A primeira integral acima já foi resolvida (ver equação (3.63)), já a segunda integral é nula, uma vez que se trata de uma integral ímpar em um intervalo simétrico ( $f_{0}$ dada por (3.59) é uma função par). Portanto, basta efetuarmos a última integral para obter a correção na viscosidade. Substituindo $f_{0}$ encontramos a seguinte expressão:

$$
-2 m \frac{\tau_{1}^{5}}{\tau_{2}^{2}} \frac{\partial u_{x}{ }^{3}}{\partial z} n\left(\frac{m}{2 \pi k_{B} T}\right)^{3 / 2}\left(\frac{m}{k_{B} T}\right)^{3} \int v_{x}^{4} v_{z}^{4} e^{\frac{-m\left(v_{x}^{2}+v_{y}^{2}+v_{z}^{2}\right)}{2 k_{B} T}} d^{3} v
$$

Efetuando uma simples integração encontramos o resultado:

$$
-18 \eta \tau_{1}^{2}\left(\frac{\tau_{1}}{\tau_{2}} \frac{\partial u_{x}}{\partial z}\right)^{2} \frac{\partial u_{x}}{\partial z}
$$

Utilizando a equação acima e (3.57) podemos escrever a componente da pressão corrigida:

$$
\mathbf{P}_{x z}=-\eta\left(1+18 \tau_{1}^{2}\left(\frac{\tau_{1}}{\tau_{2}} \frac{\partial u_{x}}{\partial z}\right)^{2}\right) \frac{\partial u_{x}}{\partial z}
$$

Comparando com a equação (2.39), encontramos que a viscosidade corrigida é escrica como

$$
\eta^{(2)}=\eta\left(1+18 \tau_{1}^{2}\left(\frac{\tau_{1}}{\tau_{2}} \frac{\partial u_{x}}{\partial z}\right)^{2}\right)
$$

onde a viscosidade $\eta$ do modelo BGK (ver (3.64)) é recuperada no limite $\tau_{2} \gg \tau_{1}$.

Diferentemente da condutividade térmica, a viscosidade define naturalmente um novo tempo característico,

$$
\frac{1}{t_{c}}=\frac{1}{\left|\frac{\partial u_{x}}{\partial z}\right|}
$$


Pela analogia com o tempo de relaxação da primeira ordem, podemos definir um comprimento característico relacionado ao gradiente de velocidade,

$$
L_{V}=\bar{v} t_{c}
$$

onde o subíndice $V$ faz referência ao gradiente de velocidades. Note que se considerarmos $t_{c} \sim \tau_{2}>\tau_{1}$, então podemos escrever

$$
\eta^{(2)}=\eta\left(1+18\left(\frac{l}{L_{V}}\right)^{4}\right)=\eta\left(1+18 K_{n_{V}}^{4}\right)
$$

onde $K_{n_{V}}$ é o número de Knudsen e o subíndice $V$ fazendo referência ao gradiente de velocidades.

Tal como a condutividade térmica de segunda ordem, vemos que a correção obtida para a viscosidade é também determinada pelo número de Knudsen.

\subsubsection{Condutividade elétrica}

Finalmente, determinaremos a condutividade elétrica de segunda ordem, supondo as mesmas condições utilizadas no cálculo da condutividade elétrica apresentado na seção (3.4.4). Assim, para um plasma colocado sob a influência de um pequeno campo elétrico, a equação de Boltzmann pode ser escrita através de uma perturbação do tipo $f=f_{0}+f_{1}$. Das equações $(3.50)$ e (4.15), podemos escrever $f_{1}$ da seguinte forma:

$$
f_{1}=-\frac{\tau_{1} e}{m} \mathbf{E} \cdot \nabla_{v} f_{0}-\frac{\tau_{1}}{\tau_{2}} \frac{\left(e \tau_{1} \mathbf{E} \cdot \nabla_{v} f_{0}\right)^{2}}{m^{2} f_{0}}-\frac{\tau_{1}^{2}}{\tau_{2}^{2}} \frac{\left(e \mathbf{E} \cdot \nabla_{v} f_{0}\right)^{3}}{m^{3} f_{0}^{2}} .
$$

Substituindo na definição de densidade de corrente dada por (3.66), encontramos a seguinte expressão:

$$
\begin{aligned}
\mathbf{J}= & -e \int_{v} \frac{e \tau_{1}}{k_{B} T} \mathbf{v} \frac{\left(\mathbf{E} \cdot \boldsymbol{\nabla}_{v} f_{0}\right)}{m} d^{3} v-e \int_{v} \frac{\tau_{1}}{\tau_{2}}\left(\frac{e \tau_{1}}{k_{B} T}\right)^{2} \mathbf{v} \frac{\left(\mathbf{E} \cdot \boldsymbol{\nabla}_{v} f_{0}\right)^{2}}{m^{2} f_{0}} d^{3} v \\
& -e \int_{v} \frac{\tau_{1}}{\tau_{2}}\left(\frac{e \tau_{1}}{k_{B} T}\right)^{2} \mathbf{v} \frac{\left(\mathbf{E} \cdot \boldsymbol{\nabla}_{v} f_{0}\right)^{3}}{m^{3} f_{0}^{2}} d^{3} v
\end{aligned}
$$

A primeira integral já foi resolvida quando encontramos a condutividade elétrica usando o modelo BGK (ver (3.67)). Usando a relação de ortogonalidade dada por (4.18), vemos que 
a segunda integral é nula. Assim, nos resta resolver a terceira integral para encontrarmos a condutividade elétrica usando a aproximação tempo de relaxação de segunda ordem. Em coordenadas esféricas e substituindo $f_{0}$ (definida em (3.11)), a integral em questão pode ser reescrita como:

$$
\frac{32 \pi}{5} n \frac{\tau_{1}^{5} e^{4}}{k_{B}^{2} T^{2} \tau_{2}^{2}}\left(\frac{m}{2 \pi k_{B} T}\right)^{3 / 2} E^{2} \mathbf{E} \int v^{6} e^{-\frac{m v^{2}}{2 k_{B} T}} d v
$$

Finalmente, efetuando a integral acima encontramos:

$$
6 \sigma\left(\frac{\tau_{1}}{\tau_{2}} \frac{e l E}{\frac{m \bar{v}^{2}}{2}}\right)^{2} \mathbf{E}
$$

A expressão final para a densidade de corrente elétrica com base no modelo de segunda ordem toma a seguinte forma:

$$
\mathbf{J}=\sigma\left(1+6\left(\frac{\tau_{1}}{\tau_{2}} \frac{e l E}{\frac{m \bar{v}^{2}}{2}}\right)^{2}\right) \mathbf{E}
$$

onde o caso linear é recuperado no limite $\tau_{2} \gg \tau_{1}$.

Comparando a equacão fenomenológica (2.44) com a equação acima, podemos escrever a condutividade elétrica para o termo de segunda ordem como:

$$
\sigma^{(2)}=\sigma\left(1+6\left(\frac{\tau_{1}}{\tau_{2}} \frac{e l E}{\bar{K}}\right)^{2}\right)
$$

onde $\bar{K}$ é a energia cinética média dos elétrons.

Da mesma forma que a para o caso da condutividade térmica, podemos definir o comprimento característico relacionado a força generalizada decorrente da perturbação. Desta forma, definimos o comprimento característico nestas condições como:

$$
\frac{1}{L_{E}}=\frac{e E}{\bar{K}}
$$

onde o subíndice $E$ faz referência ao campo elétrico atuante no processo de condução. 
Escrita em função do comprimento característico a condutividade elétrica fica:

$$
\sigma^{(2)}=\sigma\left(1+6\left(\frac{\tau_{1}}{\tau_{2}} \frac{l}{L_{E}}\right)^{2}\right)
$$

Novamente encontramos o "número de Knudsen", aqui representado por $l / L_{E}$ para nossa correção de segunda ordem. O comprimento característico relacionado com a força elétrica, evidência que, embora pequeno, diferentes intensidades de campos elétricos produzem diferentes correções.

Como foi feito no caso da condução térmica, podemos tentar definir o tempo de relaxação de segunda ordem relativo a condução elétrica. Por analogia com o tempo de relaxação de primeira ordem definimos:

$$
\tau_{2}=\frac{L_{E}}{\bar{v}}
$$

onde $\bar{v}$ é a velocidade média.

Substituindo novamente a definição de $\tau_{2}$ e a definição (4.42) em (4.41) encontramos a condutividade elétrica em função do número de Knudsen.

$$
\sigma^{(2)}=\sigma\left(1+6 K_{n_{E}}^{4}\right)
$$

onde o subíndice $E$ faz referência ao gradiente do campo elétrico e sua contribuição no cálculo perturbativo.

Encontramos aqui a correção do modelo de segunda ordem em função do número de Knudsen, constatando o fato já evidenciado na literatura, de que correções de ordem superior nos gradientes devem aparecer como potências de $K_{n_{E}}$. O caso linear é recuperado ao desprezarmos as correções de ordem superior em $K_{n_{E}}$ (3.69). De fato ,esta é uma condição sob a qual o modelo linear é tratado [3].

\subsubsection{Razão de Eucken e Correções de Segunda Ordem}

Nesta seção iremos determinar a razão de Eucken usando os resultados obtidos através do modelo de relaxação de segunda ordem. Substituindo na relação dada por (3.70) a condutividade térmica e a viscosidade dadas, respectivamente, por (4.21) e (4.32), encon- 
tramos:

$$
\Lambda^{(2)}=\Lambda \frac{1+184,8\left(\frac{\tau_{1}}{\tau_{2}} \frac{l|\nabla T|}{T}\right)^{2}}{1+18 \tau_{1}^{2}\left(\frac{\tau_{1}}{\tau_{2}} \frac{\partial u_{x}}{\partial z}\right)^{2}} .
$$

onde $\Lambda=\kappa m / k_{B} \eta$ é a razão de Eucken de primeira ordem.

Note pela equação acima que as correções na condutividade elétrica e viscosidade atuam de maneira distinta na razão de Eucken. Enquanto a primeira tende a aumentá-la, a segunda sempre tende a diminuí-la.

Uma vez que $l$ pode ser escrito em função da temperatura e os tempos característico são constantes, podemos dizer que o incremento na razão de Eucken é de origem térmica, enquanto o decremento é de origem mecânica.

\subsection{Modelo Colisional Tipo Lei de Potência}

Nesta seção vamos considerar uma segunda possibilidade de generalizar o modelo BGK, propondo uma generalização tipo lei de potência. Como foi visto na secção 3.3.4, a relaxação do sistema para o estado de equilíbrio ocorre de forma exponencial. Nossa proposta é apresentar um modelo cujo decaimento para o equilíbrio seja descrito por uma lei de potência. Como veremos, este modelo se apresentará mais geral que o decaimento exponencial, sendo este um caso particular do modelo.

A motivação desta abordagem é baseada em uma série de artigos presentes na literatura $[49,50,51]$, onde é demonstrado que modelos colisionais dados por leis de potência podem descrever o fenômeno de relaxação, além de satisfazerem o teorema $\mathrm{H}$.

O modelo lei de potência baseado na generalização do modelo BGK é escrito como:

$$
\left(\frac{\delta f}{\delta t}\right)=-\frac{f_{0}}{\tau}\left(\frac{f-f_{0}}{f_{0}}\right)^{\alpha}
$$

onde $\tau$ é o tempo de relaxação, $f_{0}$ é a função distribuição do equilíbrio e $\alpha$ a potência, definida como $\alpha \geq 1$. No caso limite $\alpha \rightarrow 1$ é recuperado o decaimento exponencial do modelo linear.

Para melhor compreender a equação (4.47) podemos resolver a equação de Boltzmann em um caso simples como feito na seção 3.3.4. Para isso supomos um plasma diluído não magnetizado em um meio ausente de gradientes espaciais e campos externos. Assim a 
equação de Boltzmann aplicada ao modelo colisional tipo lei de potência é escrita como:

$$
\frac{\partial f}{\partial t}=-\frac{f_{0}}{\tau}\left(\frac{f-f_{0}}{f_{0}}\right)^{\alpha}
$$

Definindo a função adimencional $g(\mathbf{v}, t)=\left(f(\mathbf{v}, t)-f_{0}\right) / f_{0}$, podemos escrever:

$$
\frac{\partial g}{\partial t}=-\frac{g^{\alpha}}{\tau}
$$

Resolvendo a simples integral acima encontramos:

$$
f=f_{0}\left(1+\frac{f(\mathbf{v}, 0)-f_{0}}{f_{0}}\right)\left[1-(1-\alpha) \frac{t}{\tau}\left(\frac{f_{0}}{f(\mathbf{v}, 0)-f_{0}}\right)^{1-\alpha}\right]^{\frac{1}{1-\alpha}}
$$

onde o decaimento exponencial dado pelo modelo linear é recuperado no limite $\alpha=1$.

Vamos agora tomar a seguinte definição:

$$
\tau^{\prime}(v)=\tau\left(\frac{f(\mathbf{v}, 0)-f_{0}}{f_{0}}\right)^{1-\alpha}
$$

Esta expressão para o tempo de relaxação o define como uma função da velocidade. De fato, o tempo de relaxação apresentado no modelo BGK, no qual nossa generalização é baseada, depende da velocidade, contudo ele é constante como um primeira aproximação. Aqui faremos a mesma aproximação do caso linear, assumindo $\tau^{\prime}(v) \approx \tau$ a fim de comparar a solução obtida através do modelo lei de potência com a solução encontrada a partir do modelo linear.

Na figura 4.2 fazemos uma comparação entre o decaimento exponencial do modelo linear e o decaimento proprocionado pelo modelo lei de potência para vários valores de $\alpha$.

Analisando o gráfico vemos que o decaimento proporcionado pela lei de potência aumenta o tempo necessário para o sistema chegar ao equilíbrio, desta forma propiciando um tempo de decaimento sempre maior que o previsto pelo modelo BGK (decaimento exponencial). Note que tal comportamento é exatamente o oposto do previsto pelo modelo de segunda ordem (ver figura 4.1). 


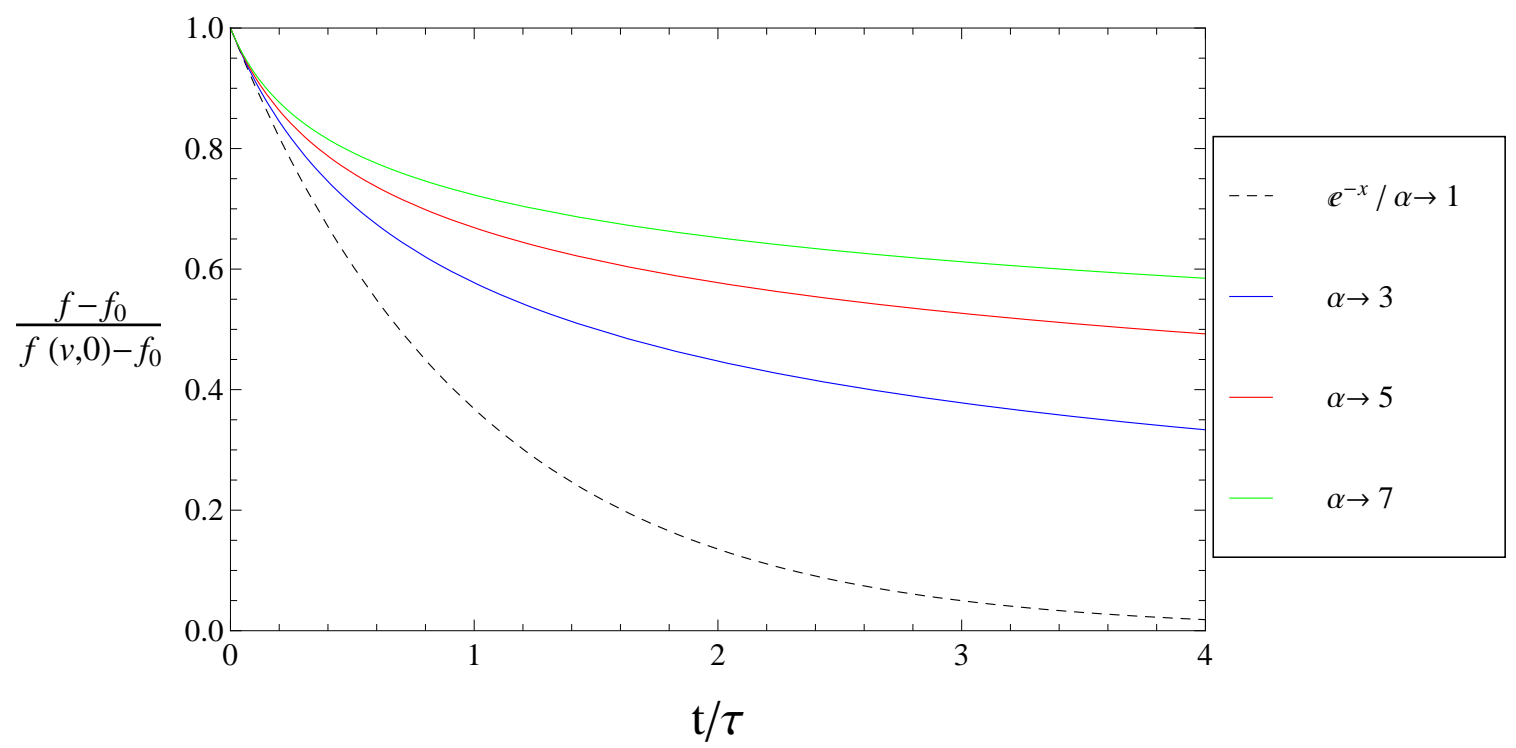

Figura 4.2: Decaimento para o equilíbrio. O gráfico mostra o decaimento em direção ao equílibrio para diversos valores de $\alpha$. O caso limite $\alpha=1$, descreve decaimento exponencial previsto pelo modelo BGK. Notamos ainda que o tempo necessário para atingir o equilíbrio termodinâmico através de uma lei de potência é sempre maior do que no caso exponencial.

\subsection{Cálculo da Condutividade Elétrica}

Nesta seção, como uma aplicação do modelo lei de potência, apresentaremos o cálculo para a condutividade elétrica. Escolhemos tal coeficiente devido à simplicidade de seu cálculo, deixando para o futuro os cálculos da condutividade térmica e da viscosidade.

Primeiramente devemos escrever a equação de Boltzmann para o modelo colisional tipo lei de potência. Para isso vamos utilizar aqui as mesmas condições apresentadas na seção (3.4.4), onde o cálculo foi realizado para o modelo linear. Nestas condições podemos escrever a equação de Boltzmann na presença de um campo elétrico orientado na direção $\hat{\mathbf{z}}$ da seguinte maneira:

$$
\frac{e E_{z}}{m} \cdot \frac{\partial f}{\partial v_{z}}=\frac{f_{0}}{\tau}\left(\frac{f-f_{0}}{f_{0}}\right)^{\alpha},
$$

onde e é a carga dos elétrons e $f_{0}$ a função de distribuição de equilíbrio local (3.11).

Considerando pequenos desvios do equilíbrio, é possível descrever a perturbação do sistema como $f=f_{1}+f_{0}$. Assim, a equação de Boltzmann perturbada se reduz para:

$$
\frac{e E_{z}}{m} \cdot \frac{\partial f_{0}}{\partial z}=\frac{f_{0}}{\tau}\left(\frac{f_{1}}{f_{0}}\right)^{\alpha}
$$

onde foi considerado $\partial f_{1} \partial v_{z} \ll \partial f_{0} / \partial v_{z}$. 
A partir da equação acima a solução geral para $f_{1}$ pode ser escrita da seguinte:

$$
f_{1}=f_{0}\left(\frac{\tau e E_{z}}{m f_{0}} \frac{\partial f_{0}}{\partial z}\right)^{1 / \alpha}
$$

Substituindo $f_{1}$ na definição de densidade de corrente elétrica (3.66), encontramos a seguinnte expressão em coordenadas esféricas:

$$
\mathbf{J}=(-1)^{1 / a} \frac{4}{3} \frac{\pi e^{2} n \tau}{k_{B} T}\left(\frac{m}{2 \pi k_{B} T}\right)^{\frac{3}{2}}\left(\frac{e E \tau}{k_{B} T}\right)^{\frac{1-\alpha}{\alpha}} E \int_{v} v_{z}\left(v_{z} E_{z}\right)^{1 / a} e^{\frac{-m\left(v_{x}^{2}+v_{y}^{2}+v_{z}^{2}\right)}{2 k_{B} T}} d^{3} v
$$

onde foi feita a divisão por 3 da equação devido a isotropia do plasma e as integrais triplas que se estendem de $-\infty$ até $\infty$.

Efetuando a integral encontramos o seguinte resultado:

$$
J_{z}=-(-1)^{\frac{1}{\alpha}} \sigma \frac{4}{3} \frac{\Gamma\left(2+\frac{1}{2 \alpha}\right)}{\sqrt{\pi}}\left(\frac{e l E}{\frac{1}{2} m \bar{v}^{2}}\right)^{\frac{1-\alpha}{\alpha}} E_{z}
$$

onde $\sigma$ a condutividade elétrica encontrada através do modelo linear e o caso padrão é recuperado tomando $\alpha=1$ (ver (3.68)).

Comparando a equação cima coma equação fenomenológica (2.44), podemos definir a condutividade elétrica resultande do modelo colisional tipo lei de potência como:

$$
\sigma^{(\alpha)}=-(-1)^{\frac{1}{\alpha}} \sigma \frac{4}{3} \frac{\Gamma\left(2+\frac{1}{2 \alpha}\right)}{\sqrt{\pi}}\left(\frac{e l E}{\bar{K}}\right)^{2 \frac{1-\alpha}{\alpha}}
$$

onde $\bar{K}=m \bar{v}^{2} / 2$ é a energia cinética média dos portadores de carga.

Vemos da equação acima que determinados valores de $\alpha$ implicam valores imaginários para a condutividade elétrica. Este resultado não é permitido devido á relação dos coeficientes de transporte com a segunda lei da termodinâmica. Portanto, devemos escolher adequadamente $\alpha$ a fim de eliminar estes casos que não possuem significado físico. Para isso escolhemos $\alpha=2 n+1$ com $n=0,1,2, \ldots$, ou seja, um número inteiro positivo ímpar. Com esta hipótese a equação (4.57) pode ser reescrita como

$$
\sigma^{(n)}=\sigma \frac{4}{3} \frac{\Gamma\left(2+\frac{1}{4 n+2}\right)}{\sqrt{\pi}}\left(\frac{e l E}{\bar{K}}\right)^{-\frac{4 n}{2 n+1}}
$$

Pela equação acima observamos que a correção é dada em função da força generali- 


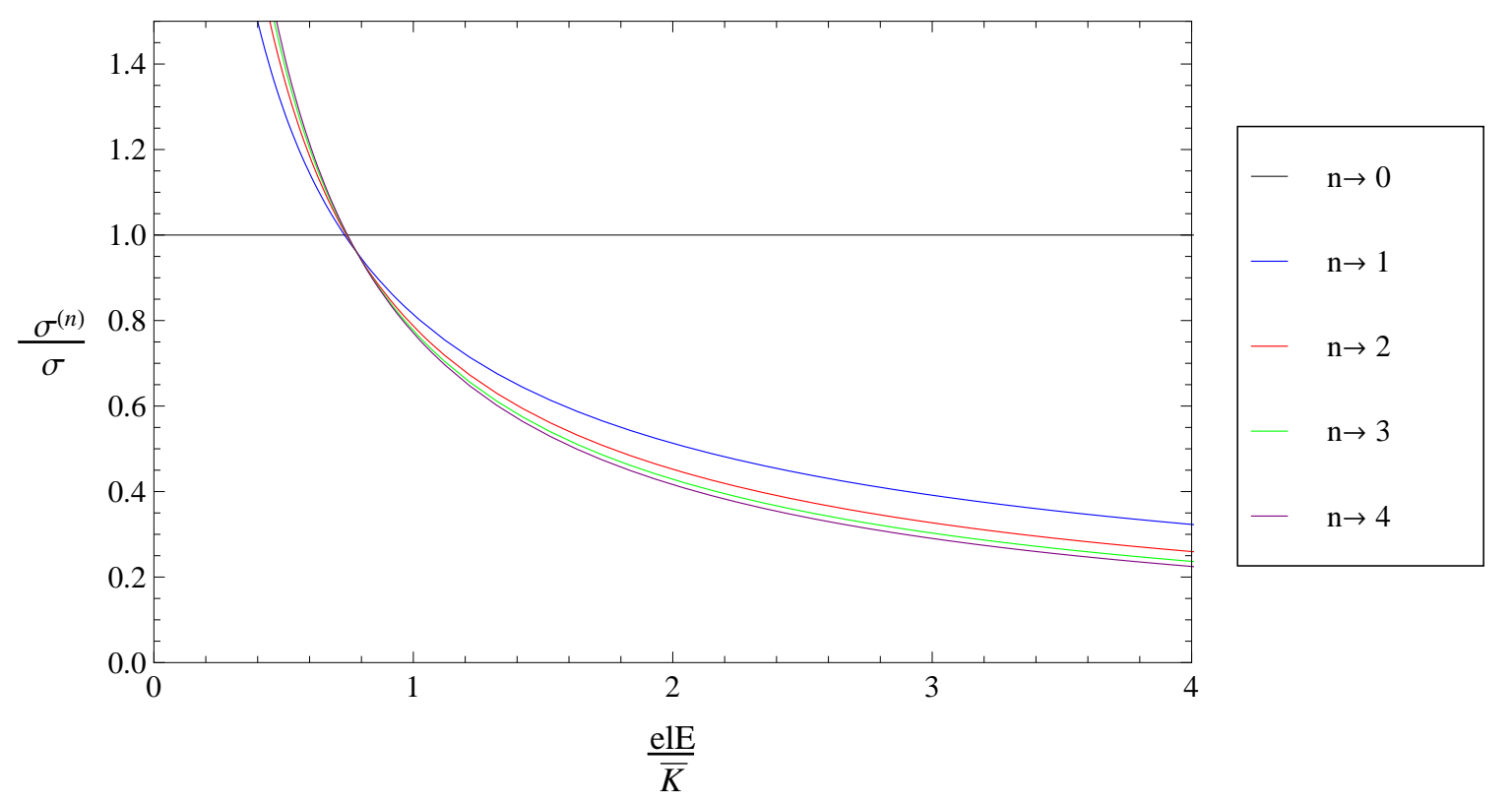

Figura 4.3: Condutividade Elétrica Segundo a Lei de Potências. O gráfico mostra o comportamento da condutividade elétrica estabelecida através do modelo colisional lei de potências.

zada, no caso a força elétrica, onde a correção é uma lei de potência que multiplica a condutividade elétrica obtida no caso linear. Na figura 4.3 mostramos o comportamento da condutividade elétrica para vários valores de $\alpha$.

Da mesma forma que foi feito para a correção de segunda ordem, utilizando a definição do número de Knudsen e o comprimento característico relacionado ao campo elétrico $E_{z}$ (4.42), podemos escrever a condutividade elétrica como:

$$
\sigma^{(\alpha)}=\sigma \frac{4}{3} \frac{\Gamma\left(1+\frac{1}{4 n+2}\right)}{\sqrt{\pi}}\left(\frac{l}{L_{E}}\right)^{-\frac{4 n}{2 n+1}}=\sigma \frac{4}{3} \frac{\Gamma\left(1+\frac{1}{4 n+2}\right)}{\sqrt{\pi}}\left(K_{n_{E}}\right)^{-\frac{4 n}{2 n+1}}
$$

O resultado acima mostra que a correção é obtida em função de $K_{n_{E}}$. De fato, o modelo lei de potência é de ordem superior ao modelo BGK (para $\alpha>1$ ), portanto, como no caso do modelo de segunda ordem (ver seção 4.3) é de se esperar a presença de $K_{n_{E}}$. 
Capítulo 5

\section{Conclusões e Perspectivas}

Neste trabalho estudamos dois modelos colisionais alternativos baseados na generalização do modelo BGK [14]. Inicialmente, propomos um modelo tempo de relaxação de segunda ordem, através do qual introduzimos um tempo de relaxação adicional $\tau_{2}$. Em seguida, inspirados em resultados relacionados com a estatística de Tsallis [49, 50], generalizamos o modelo BGK através de uma lei de potência. Nas duas generalizações, o modelo BGK padrão é recuperado como um caso particular.

Começamos o capítulo 4 investigando as consequências do modelo tempo de relaxação de segunda ordem. Para isto, o modelo foi aplicado em um caso bastante simples, visando facilitar a compreensão física do novo termo adicionado. Nesse sentido, tratamos a relaxação do sistema para o equilíbrio na ausência de gradientes e campos externos. Vimos pela equação (4.10) que o termo de segunda ordem acelera o processo de decaimento para o estado de equilíbrio diminuindo, portanto, o tempo de total de evolução necessário para que se alcance tal estado. Este resultado pode ser entendindo considerando que o termo de segunda ordem adiciona efeitos colisionais anteriormente desprezados. A fonte de entropia será maior e consequentemente deverá haver uma conversão maior da energia do movimento microscópico em calor ou trabalho.

Na seção 4.3 aplicamos nosso modelo para o cálculo de alguns coeficientes de transporte, a saber: a condutividade térmica $(\kappa)$, a viscosidade $(\eta)$ e a condutividade elétrica $(\sigma)$. Como esperado, as correções obtidas para $\kappa, \eta$ e $\sigma$ foram de ordem $\tau_{1} / \tau_{2}$, onde o caso

padrão (modelo BGK) é recuperado no limite $\tau_{2} \gg \tau_{1}$. Embora, o número de Knudsen obtido seja na quarta ordem (ver as expressões em (4.26), (4.35) e (4.45)), isto se deve á necessidade de expandirmos a solução $f_{1}$ até a terceira ordem.

As correções obtidas para os coeficientes de transporte se mostraram proporcionais ás 
forças termodinâmicas generalizadas que induzem o fenômeno de transporte. Desta forma, tais perturbações se tornam parte da descrição do problema, isto é, na ordem considerada os coeficientes de transporte deixam de ser apenas uma característica do meio e passam a depender também dos fenômenos que ocorrem no mesmo. Vemos pelas equações (4.21), (4.32) e (4.41) que as correções da condutividade térmica, $\kappa(|\nabla T|, T)$ e a condutividade elétrica $\sigma(E, T)$ ) são funções da temperatura e da força perturbativa que induz o fenômeno de transporte, enquanto a viscosidade, $\eta\left(\left|\partial u_{z} / \partial z\right|\right)$, é apenas função do gradiente de velocidade. Outro resultado interessante é a presença do número de Knudsen, $K_{n}$, introduzido através das definições dos comprimentos característicos $L_{T}, L_{V}$ e $L_{E}$, definindo os comprimentos característicos das forças termodinâmicas generalizadas.

No fim desta seção calculamos a razão de Eucken através do modelo de segunda ordem $\left(\Lambda^{(2)}=\kappa^{(2)} m / k_{B} \eta^{(2)}\right)$. Pela equação (4.46) vemos que as correções para a condutividade térmica e para a viscosidade atuam de maneira distinta. Enquanto a primeira contribui para aumentar o valor de $\Lambda^{(2)}$, a segunda o diminui. Desta forma vemos que a correção na relação de Eucken depende da importância relativa dos fenômenos térmicos e mecânicos. Em outra palavras, quando os fenômenos térmicos forem mais importantes, esperamos um aumento em $\Lambda^{(2)}$; em contrapartida, sendo fenômenos mecânicos mais importantes esperamos uma diminuição da razão de Eucken. Embora a correção permita o crescimento, ou decrescimento, sabemos que os valores possíveis de $\Lambda^{(2)}$ estão dentro de um faixa experimentalmente determinada. Assim, vemos que o modelo é aplicável apenas para um determinado intervalo, o que deverá limitar naturalmente o alcance da aproximação adotada.

Na seção 4.5 discutimos o modelo colisional tipo lei de potência. Como anteriormente, iniciamos a seção com uma discussão simplificada do modelo, inspirado na teoria nãoextensiva e recentes trabalhos neste contexto [49, 50, 52]. Nesta discussão simplificada, adotamos o decaimento para o equilíbrio desprezando todos os gradientes. Como se trata de um lei de potência, vimos que a solução neste caso é também uma lei de potência, onde o modelo BGK é um caso particular $(\alpha=1)$. O decaimento para o equilíbrio obtido pelo modelo em questão se mostrou mais lento quando comparado aos proporcionados pelos modelos exponencial e de segunda ordem. De fato, uma função do tipo lei de potência decresce mais lentamente que uma do tipo exponencial (ver figuras 4.1 e 4.2).

Como uma aplicação do modelo calculamos a condutividade elétrica para um plasma 
não magnetizado e diluído. A correção obtida neste contexto se apresenta como o produto de três termos: a condutividade elétrica obtida através do modelo BGK, um coeficiente numérico dependemte de $\alpha$ e uma lei de potência no termo elE $/ \bar{K}$. É interessante notar que esta forma se assemelha á forma do chamado Tensor de Piola Kirchhoff, onde o tensor da deformação é proporcional ao determinante do gradiente de perturbação [53]. Este tipo de tensor é relacionado á descrição de grandes deformações em corpos sólidos contínuos que impossibilitam o uso do tensor de Cauchy. Por analogia podemos inferir, a partir da forma do coeficiente de transporte, que nossa abordagem através do modelo lei de potência pode vir a ser utilizada na descrição de fenômenos de transporte com perturbações (forças termodinâmicas e generalizadas) moderadamente intensas.

A partir da definição do comprimento característico $L_{E}$ para a força termodinâmica generalizada (força elétrica), determinamos também a correção para a condutividade elétrica como uma lei de potência em $K_{n}$. De fato, é esperada a presença de $K_{n}$ na correção, uma vez que se trata de uma correção de ordem superior ao caso obtido pelo modelo BGK, o caso linear (ver $[3,8]$ ). Como $L_{E}$ diminue á medida que $e E / \bar{K}$ aumenta, vemos que para perturbações moderadas nosso modelo prevê uma condutividade elétrica menor. Também é importante resaltar que nosso modelo determina a condutivdade elétrica como função da temperatura e do campo elétrico $(\sigma(E, T))$ (ver equação $(4.59))$, além do parâmentro livre $\alpha$.

Os resultados apresentados aqui ainda podem ser expandidos e aplicados nos casos em que o modelo BGK falha. Os exemplos já citados, a saber, ondas de choque, ondas de som ultrasonicas, magnetoplasmas em campos magnéticos intensos, etc [8]. Como uma aplicação direta, indicamos os cálculos da condutividade térmica e viscosidade através do modelo lei de potência (estas soluções analíticas devem aparecer em nossos futuros trabalhos). Deixamos também como sugestão o cálculo da viscosidade volumar e do coeficiente de difusão para ambos os modelos. Da mesma forma, é bastante interessante o confronto de dados experimentais para a razão de Eucken com a previsão obtida através do modelo de segunda ordem. Outras linhas a serem exploradas são a conexão da teoria não-extensiva com modelo tipo lei de potência e a adição de termos de ordem superior no modelo colisional de segunda ordem (4.1). Outro problema que também deverá ser tratado no futuro é a existência de uma contraparte relativística para os modelos colisionais alternativos discutidos nesta dissertação. 
Apêndice 



\section{Apêndice A}

\section{Elementos de Volume no Espaço de Fase - Invariância}

Neste apêndice discutiremos a invariância (sob transformação de coordenadas) dos elemento de volume no espaço de fase. Pela teoria da matriz jacobiana, sabemos que sob uma mudança de variáveis, a transformação do elemento de volume no espaço de fase é obtida a partir do determinante da matriz jacobiana. Desta forma podemos escrever:

$$
d^{3} r d^{3} v=|J| d^{3} r^{\prime} d^{3} v^{\prime}
$$

A matriz Jacobiana é definida da seguinte maneira [12]:

$$
J=\frac{\partial\left(\mathbf{r}^{\prime}, \mathbf{v}^{\prime}\right)}{\partial(\mathbf{r}, \mathbf{v})}=\frac{\partial\left(x^{\prime}, y^{\prime}, z^{\prime}, v_{x}^{\prime}, v_{y}^{\prime}, y_{z}^{\prime}\right)}{\partial\left(x, y, z, v_{x}, v_{y}, v_{z}\right)}
$$

Explicitamente a matriz pode ser escrita como [54]:

$$
J=\left(\begin{array}{cccc}
\partial x^{\prime} / \partial x & \partial y^{\prime} / \partial x & \ldots & \partial v_{z}^{\prime} / \partial x \\
\partial x^{\prime} / \partial y & \partial y^{\prime} / \partial y & \ldots & \partial v_{z}^{\prime} / \partial y \\
\vdots & \vdots & \vdots & \vdots \\
\partial x^{\prime} / \partial v_{z} & \partial y^{\prime} / \partial v_{z} & \ldots & \partial v_{z}^{\prime} / \partial v_{z}
\end{array}\right)
$$

Vamos supor também que a força externa é separável como uma soma de duas parcelas, sendo uma parte independente da velocidade e outra independende da posição $(\mathbf{F}=\mathbf{F}(\mathbf{r})+$ $\mathbf{F}(\mathbf{v})$ ). Neste caso, podemos escrever as seguintes relações:

$$
\begin{aligned}
\frac{\partial x_{i}^{\prime}}{\partial x_{j}}=\delta_{i j} & , \quad \frac{\partial v_{i}^{\prime}}{\partial x_{i}}=\frac{1}{m} \frac{\partial F\left(\mathbf{r}_{i}\right)}{\partial x_{j}} \\
\frac{\partial x_{i}^{\prime}}{\partial v_{j}}=\delta_{i j} d t & , \quad \frac{\partial v_{i}^{\prime}}{\partial v_{j}}=\delta_{i j}+\frac{1}{m} \frac{\partial F(\mathbf{v})}{\partial v_{j}} .
\end{aligned}
$$


Considerando as transferências acima, vemos que a matriz pode ser reescrita da seguinte forma:

$$
J=\left(\begin{array}{ll}
(J)_{1} & (J)_{2} \\
(J)_{3} & (J)_{4}
\end{array}\right)
$$

onde $(J)_{i} \operatorname{com} i=1,2,3,4$ são as seguintes matrizes:

$$
\begin{gathered}
(J)_{1}=\left(\begin{array}{lll}
1 & 0 & 0 \\
0 & 1 & 0 \\
0 & 0 & 1
\end{array}\right) \\
(J)_{2}=\frac{d t}{m}\left(\begin{array}{lll}
\partial F_{x}^{\prime} / \partial x & \partial F_{y}^{\prime} / \partial x & \partial F_{z}^{\prime} / \partial x \\
\partial F_{x}^{\prime} / \partial y & \partial F_{y}^{\prime} / \partial y & \partial F_{z}^{\prime} / \partial y \\
\partial F_{x}^{\prime} / \partial z & \partial F_{y}^{\prime} / \partial z & \partial F_{z}^{\prime} / \partial z
\end{array}\right) \\
(J)_{4}=\frac{d t}{m}\left(\begin{array}{lll}
1 & 0 & 0 \\
\partial F_{x}^{\prime} / \partial v_{x} & \partial F_{y}^{\prime} / \partial v_{x} & \partial F_{z}^{\prime} / \partial v_{x} \\
\partial F_{x}^{\prime} / \partial v_{y} & \partial F_{y}^{\prime} / \partial v_{y} & \partial F_{z}^{\prime} / \partial v_{y} \\
\partial F_{x}^{\prime} / \partial v_{z} & \partial F_{y}^{\prime} / \partial v_{z} & \partial F_{z}^{\prime} / \partial v_{z}
\end{array}\right)
\end{gathered}
$$

Calculando o determinante de $J$ e desprezando termos de ordem maior ou igual a $d t$ obtemos $|J|=1$, mostrando que a relação (A.1) expressa a invariância matemática dos elementos de volume no espaço:

$$
d^{3} r d^{3} v=d^{3} r^{\prime} d^{3} v^{\prime}
$$




\section{Apêndice B}

\section{Decomposição do Produto Tensorial (Tensor de Viscosidade)}

Neste apêdice discutiremos a separação do produto interno entre os tensores $\boldsymbol{\Pi}$ e $\boldsymbol{\nabla} \mathbf{v}$, utilizada no capítulo 2 (ver (2.19)):

$$
\Pi: \nabla \mathbf{v}
$$

Vamos agora escrever os tensores $\boldsymbol{\Pi}$ e $\boldsymbol{\nabla} \mathbf{v}$ (matrizes $3 \times 3$ ) como a soma de suas componentes na diagonal e fora da diagonal. Nesta decomposição o tensor $\Pi$ é:

$$
\Pi=\Pi I+\stackrel{\circ}{\Pi}
$$

onde $I_{i j}=\delta_{i j}$ (delta de kronecker) é a matriz identidade. As matrizes $\Pi$ e $\Pi$ são definidos por:

$$
\begin{aligned}
\Pi & =\frac{1}{3} \boldsymbol{\Pi}: \mathbf{I}=\frac{1}{3} \sum_{\gamma=1}^{3} \Pi_{\gamma \gamma} . \\
\stackrel{\circ}{\boldsymbol{\Pi}} I & =\sum_{\gamma=1}^{3} \stackrel{\circ}{\Pi}_{\gamma \gamma}=0 .
\end{aligned}
$$

Similarmente, o tensor $\boldsymbol{\nabla} \mathbf{v}$ pode ser escrito como:

$$
\boldsymbol{\nabla} \mathbf{v}=\frac{1}{3}(\boldsymbol{\nabla} \cdot \mathbf{v} \mathbf{I}+\stackrel{\circ}{\nabla} \mathbf{v})
$$


onde o traço de $\boldsymbol{\nabla} \mathbf{v}$ é dado por:

$$
\boldsymbol{\nabla} \cdot \mathbf{v}=\boldsymbol{\nabla} \mathbf{v}: \mathbf{I}=\sum_{\beta=1}^{3} \frac{\partial v_{\beta}}{\partial x_{\beta}}
$$

enquanto o traço de $\stackrel{\circ}{\nabla} \mathbf{v}$ é nulo:

$$
\stackrel{\circ}{\nabla} \mathbf{v}: \mathbf{I}=\sum_{\gamma=1}^{3}\left(\frac{\partial v_{\gamma}}{\partial x_{\gamma}}-\frac{1}{3} \sum_{\beta} \frac{\partial v_{\gamma}}{\partial x_{\beta}}\right)=0 .
$$

Substituindo as equações (B.2), (B.4), (B.5) e (B.7) o produto escalar (B.1) pode ser escrito como:

$$
\Pi: \nabla \mathrm{v}=\stackrel{\circ}{\Pi}: \stackrel{\circ}{\nabla} \mathrm{v}+\Pi \nabla . \mathrm{v}
$$

O tensor $\stackrel{\circ}{\nabla} \mathbf{v}$ pode ainda ser separado em uma parte simétrica e uma anti-simétrica:

$$
\stackrel{\circ}{\nabla} \mathbf{v}=(\stackrel{\circ}{\nabla} \mathbf{v})^{s}+(\stackrel{\circ}{\nabla} \mathbf{v})^{a}
$$

com

$$
\begin{array}{r}
(\stackrel{\circ}{\nabla} \mathbf{v})_{\beta \gamma}^{s}=\frac{1}{2}\left(\frac{\partial v_{\gamma}}{\partial x_{\beta}}+\frac{\partial v_{\gamma}}{\partial x_{\beta}}\right)-\frac{1}{3} \delta_{\beta \gamma} \sum_{\xi=1}^{3} \frac{\partial v_{\xi}}{\partial x_{\xi}}, \quad(\gamma, \beta=1,2,3) \\
(\stackrel{\circ}{\nabla})_{\gamma \beta}^{a}=\frac{1}{2}\left(\frac{\partial v_{\gamma}}{\partial x_{\beta}}-\frac{\partial v_{\beta}}{\partial x_{\gamma}}\right) .
\end{array}
$$

Usando (B.9) em (B.8) econtramos:

$$
\Pi: \nabla \mathbf{v}=\stackrel{\circ}{\Pi}:(\stackrel{\circ}{\nabla} \mathbf{v})^{s}+\Pi \nabla \cdot \mathbf{v}
$$

Onde foi utilizada a propriedade do produto interno duplo (produto escalar) entre um tensor simétrico e um anti-simétrico ser nula. 
Apêndice $\mathrm{C}$

\section{Equações de Balanço}

Será apresentado aqui uma breve discussão a respeito das chamadas equações de balanço. Para isso usaremos como base o primeiro capítulo do livro Thermodynamics Theory of Structure, Stability and Fluctuations, publicado por Glansdorff e Prigogine [55]. Considerando um sistema de volume $V$ e limitado pela superfície $\Omega$, a quantidade $I(t)$ é dada pela integral que se extende sobre todo o sistema $V$ :

$$
I(t)=\int_{V} f d V
$$

onde foi considerado que a superfície $\Omega$ está em repouso.

Pela nomenclatura usual da termodinâmica $I(t)$ é denominada uma quantidade extensiva como, por exemplo, a energia ou a massa [55]. Já a quantidade designada por $f$ é conhecida como uma quantidade intensiva do sistema, isto é, não depende do sistema como um todo. No caso mais geral $f$ pode depender da posição e do tempo, ou seja, $f$ é uma função de $x, y, z$ e $t$. Isto corresponde a uma densidade por unidade de volume associada a $I(t)$ que é representada pela derivada funcional:

$$
f \equiv \frac{\delta I}{\delta V}
$$

É possível distinguir entre dois mecanismos para a variação temporal de $I(t)$ de modo que escrevemos:

$$
\frac{\partial I(t)}{\partial t}=P[I]+\Phi[t]
$$

O primeiro termo da equação corresponde a produção por unidade de tempo da quan- 
tidade $I$ no interior do sistema. Podemos escrever este termo como:

$$
P[I]=\int_{V} \sigma[I] d V
$$

onde $\sigma[I]$ denota o termo de fonte da quantidade $I$ por unidade de tempo.

O segundo termo da equação (C.3) representa o fluxo da quantidade $I$ que atravessa a superfície $\Omega$ :

$$
\Phi[T]=\int_{\Omega} j_{n}[I] d \Omega
$$

onde $j_{n}[I]$ é a densidade de fluxo associado com $I$ projetada no interior do volume $V$ e na direção normal a superfície. Por simplicidade a partir de agora apenas usaremos fluxo ao invés de densidade de fluxo. Ainda é importante salientar que o fluxo $\mathbf{j}$ projetado na direção normal é o fluxo total composto por dois termos, isto é, o fluxo é formado duas partes distintas. As duas partes que formam o fluxo são o fluxo convectivo $\mathbf{j}_{\text {conv }}$ e o fluxo condutivo $\mathbf{j}_{\text {cond }}$, sendo que o segundo pode ocorrer mesmo com o sistema em equilíbrio. Desta forma podemos escrever que o fluxo total é dado por:

$$
\mathbf{j}=\mathbf{j}_{\text {conv }}+\mathbf{j}_{\text {cond }}=\mathbf{j}_{\text {cond }}+f \mathbf{v}
$$

Em geral os termos de fluxo de fonte podem ser positivos ou negativos. Usando as equação (C.4) e (C.5) em (C.3) encontramos a chamanda equação de balanço correspondente a variável extensiva $I$ :

$$
\frac{\partial I}{\partial t}=\int_{V} \sigma[I] d V+\int_{\Omega} j_{n}[I] d \boldsymbol{\Omega}
$$

Vemos portanto que a variação temporal de $I$ pode ser decomposta em duas integrais, sendo uma de superfície e outra de volume. Note que embora sendo uma quantidade escalar, poderíamos fazer um tratamento semelhante para uma grandeza vetorial. Para isso precisaríamos de uma equação de balanço para cada componente do vetor.

A igualdade (C.6) tem de ser válida independente do volume $V$, portanto a aplicação do teorema de Gauss nos leva a equação de balanço na forma local:

$$
\frac{\partial f}{\partial t}=\sigma[I]-\nabla \cdot \mathbf{j}[I]
$$

Uma das vantagens desta formulação é facilidade com que podemos obter as equações 
de conservação. Isto é feito pela simples imposição do termo de fonte, $\sigma(I)$, ser nulo. Neste caso, obtemos o tradicional resultado, algumas vezes referido como equação da continuidade:

$$
\frac{\partial f}{\partial t}+\nabla . \mathbf{J}[I]=0
$$




\section{Referências Bibliográficas}

[1] A. J. M. Spencer, "Mechanics of Continuum", Dover, Mineola (2004).

[2] L. D. Landau \& E. M. Lifshitz, "Theory of Elasticity", Pergamon Press, London (1959).

[3] L. D. Landau \& E. M. Lifshitz, "Physical Kinetics", Elsevier, New York (1981).

[4] L. D. Landau \& E. M. Lifshitz, "Fluid Mechanics", Pergamon Press, Oxford (1959).

[5] J. A. S. Lima, R. Silva \& J. Santos, Phys. Rev. E, 61, 3260 (2000).

[6] J. A. S. Lima \& R. E. de Souza, Physica A 350, 303 (2005).

[7] S. Chapman \& T. G. Cowling, "The Mathematical Thoery of Non-Uniform Gases", Cambridge University Press, Cambridge (1970).

[8] L. C. Woods, "An Introduction to Kinetic Theory of Gases and Magneto Plasmas", Oxford UP, New York (1993).

[9] E. H. Kennard, "Kinetic Theory of Gases", McGraw-Hill Book Company, New York (1938).

[10] A. Sitenko \& V. Malnev, "Plasma Physics Theory", Chapman \& Hall, Cornwall (1995).

[11] C. J. Thompson, "Mathematical Statistical Mechanics", The Macmillan Company, New York (1972).

[12] J. A. Bittencourt, "Fundamentals of Plasma Physics", Abaeté Cop. Gráfica Ltda, São Paulo (1995).

[13] V. S. Galnin \& V.A. Zharov, J. Appl. Maths Mechs 68, 1 (2004). 
[14] P. L. Bhatnagar, E. P. Gross \& M. Krook, Phys. Rev. 94, 511 (1954).

[15] E. P. Gross \& E. A. Jackson, Phys. Fluid. 2, 4 (1959).

[16] C. Tsallis, J. Stat. Phys. 52, 479 (1988).

[17] R. Silva, A. R. Plastino \& J. A. S. Lima, Phys. Lett. A 249, 401 (1998).

[18] J. A. S. Lima, R. Silva \& A. R. Plastino, Phys. Rev. Lett. 86, 2938 (2001).

[19] J. R. Bezerra, R. Silva \& J. A. S. Lima, Physica A 322, 256 (2003).

[20] A. Eucken, Phys. Z. 14, 324 (1913).

[21] D. S. Oliveira, I. Baranov \& J. A. S. Lima, "Time Relaxation Approximation and q-Maxwellian Distribuition", 2013 (em preparação).

[22] D. S. Oliveira, J. A. S Lima \& I. Baranov, "Extended BGK Method: The Kinetic Nonlinear Relaxation Time Approximation", 2013 (em preparação).

[23] W. Thomson (Lord Kelvin), Proc. Roy. Soc. Edinburgh 3, 225 (1854); Ibid. Trans. 21, I 123 (1857).

[24] S. R. de Groot \& P. Mazur, "Non-equilibrium Thermodynamics", Dover, New York (1984).

[25] L. Onsager. Phys. Rev. 37, 405 (1931); 38, 2265 (1931).

[26] I. Prigogine, "Etude thermodynamique des phénomènes irréversibles", Dunod, Paris \& Desoer, Liège (1947).

[27] I. Gyarmati, "Non-equilibirum Thermodynamics: Field Theory and Variational Principles", Springer - Verlag, Berlin Heidelberg, New York (1970).

[28] J.R. Reitz, F. M. Milford \& R. W. Christy, "Fundamentos da Teoria Eletromagnética", Campus, Rio de Janeiro (1993).

[29] D. Bernoulli \& J, Bernoulli, "Hydrodynamics \& Hydraulics", Dover, Mineola (2005).

[30] J. C. Maxwell, "Illustrations of the dynamical theory of gases", Phil. Mag. Ser. 4 19, 19 (1860). 
[31] L. Boltzmann, Lecture on Gas Theory, Dover Publications (1909).

[32] R. Reif, "Fundamentals of Statistical and Thermal Physics", McGraw-Hill, New York (1965).

[33] J. R. B. Silva, "Teoria Cinética Não-Extensiva: Coeficientes de Transporte e Outras Aplicações Físicas, Natal (2005).

[34] N. A. Krall \& A. W. Trivelpiece, "Principles of Plasma Physics", McGraw-Hill, New York (1973).

[35] G. M. Kremer, "Uma Introdução À Equação de Boltzmann”, EDUSP, São Paulo (2005).

[36] J. C. Maxwell, Phil . Trans. Roy. Soc. 157, 49 (1867).

[37] V. M. Kenkre, Phys. Letter 70A, 381 (1979)

[38] D. I. Pikulin, C. Y Hou, \& C. W. J. Beenakker Phys. Rev. B 84, 035133 (2011)

[39] I. V. Gopich \& A. Szabo, J. Chem. Phys. 117, 507 (2002)

[40] H. A. Lorentz, Arch. Néerl.10, 336 (1905).

[41] D. Hilbert, Grundzüge einer allgemeinen Theorie der linearen Intergralgleichungen. (Chelsea Pub. Co., New York, 1953).

[42] D. Enskog, Phys. Zeit 12. 56 \& 533 (1911).

[43] S. Chapman, Phil. Trans. Roy. Soc. A 217. 115 (1916).

[44] D. Burnett, Proc. London Math. Soc. 39, 275 (1935).

[45] H. Grad, Comm. Pure and Appl. Math 2, 311 (1949).

[46] K. Huang, "Statistical Mechanics", John Willey \& Sons, Cingapura (1987).

[47] Y. Zhu, J. Phys. D: Appl. Phys. 32, 2169 (1999).

[48] W. E. Boyce \& R. C. Diprima, "Elementary differential Equations and Boundary Value Problems", Jonh Wiley \& Sons, New York (1969).

[49] J. A. S Lima, R. Silva \& A. R. Plastino, Phys. Lett. A 322, 256 (2002). 
[50] R. Silva Jr., A. R. Plastino \& J. A. S Lima, Phys. Lett. A 249, 401 (1998)

[51] J. A. S Lima, R. Silva \& A. R. Plastino, Phys. Rev. Lett. 86, 14 (2001).

[52] C. Tsallis, J. Stat. Phys. 63, 479 (1971).

[53] F.S. Buezas, M.B. Rosales \& C.P. Filipich, Int. Jour. Mec. Sc. 52, 777 (2010).

[54] G. B. Arfken, "Física matemática : métodos matemáticos para engenharia e física", Campus/Elsevier, Rio de Janeiro (2007)

[55] P. Glansdorff \& I.Prigogine, "Thermodynamic Theory of Structure, Stability and Fluctuations", Wiley-Interscience, London (1971). 